\title{
Orbital Landau level dependence of the fractional quantum Hall effect in quasi-two dimensional electron layers: finite-thickness effects
}

\author{
Michael R. Peterson ${ }^{1}$, Th. Jolicoeur ${ }^{2}$ and S. Das Sarma ${ }^{1}$ \\ ${ }^{1}$ Condensed Matter Theory Center, Department of Physics, \\ University of Maryland, College Park, MD 20742, USA and \\ ${ }^{2}$ Laboratoire de Physique Théorique et Modèles Statistiques, \\ Université Paris-Sud, 91405 Orsay Cedex, France
}

\begin{abstract}
The fractional quantum Hall effect (FQHE) in the second orbital Landau level at evendenominator filling factor $5 / 2$ remains enigmatic and motivates our work. We theoretically consider the effect of the quasi-2D nature of the experimental fractional quantum Hall system on a number of FQH states (filling factors $1 / 3,1 / 5$, and 1/2) in the lowest, second, and third orbital Landau levels (LLL, SLL, and TLL, respectively) by calculating the wavefunction overlap, as a function of quasi-2D layer thickness, between the exact ground state of a model Hamiltonian and the consensus variational ansatz wavefunctions, i.e., the Laughlin wavefunction for $1 / 3$ and $1 / 5$ and the Moore-Read Pfaffian wavefunction for $1 / 2$. Using large numerical overlap as a stability, or FQHE robustness, criterion we find that the FQHE does not occur in the TLL (for any quasi-2D layer thickness), is the most robust for zero thickness (strict 2D limit) in the LLL for $1 / 3$ and $1 / 5$ and for $11 / 5$ in the SLL, and is the most robust at finite-thickness (4-5 magnetic lengths) in the SLL for the mysterious even-denominator $5 / 2$ state and the presumably more conventional $7 / 3$ state. We do not find any FQHE at 1/2 in the LLL for any thickness for the quasi-2D models considered in our work. Furthermore, we examine the orbital effects of a non-zero in-plane (parallel) magnetic field finding that its application effectively reduces the quasi-2D layer thickness and, therefore, could destroy the FQHE at 5/2 and $7 / 3$, while enhancing the FQHE at $11 / 5$, in the SLL. The in-plane field also enhances the LLL FQHE states by making the quasi-2D system more purely 2D. The in-plane field effects could thus be qualitatively different in the LLL and the SLL by virtue of magneto-orbital coupling through the finite thickness effect. Using exact diagonalization on the torus geometry, we show the appearance of the characteristic threefold topological degeneracy expected for the Pfaffian state. This signature is enhanced by nonzero thickness corroborating our findings from overlap calculations. Our results have ramifications for wavefunction engineering, opening the possibility of creating an optimal experimental system where the 5/2 FQHE state is more likely described by the Moore-Read Pfaffian state with obvious applications in the burgeoning field of fault-tolerant topological quantum computing.
\end{abstract}

PACS numbers: 73.43.-f, 71.10.Pm

\section{INTRODUCTION}

The fractional quantum Hall effect (FQHE), discovered in $1982^{1}$, is a quintessentially strongly correlated quantum phenomenon where electrons in a two-dimensional (2D) electron system condense into a strongly interacting incompressible quantum fluid ground state $^{2.3}$ with fractionally charged quasiparticle excitations which obey anyonic +5.56 , rather than ordinary fermionic or bosonic, quantum statistics. The FQHE occurs at low temperatures in clean (high mobility) 2D semiconductor structures under the influence of a strong external magnetic field applied normal to the 2D plane of confinement of the electron layer. The subject has been studied extensively during the last 25 years, and reviews $7.8,9$ can be found in the literature.

In this article we provide a detailed numerical theoretical study of the orbital Landau level (LL) dependence of the FQHE, emphasizing the relative importance of the quasi-2D layer width, i.e., the "finite-thickness effect", of the electron system transverse to the plane of confinement in the lowest three LLs. In the non-interacting $2 \mathrm{D}$ system, taken here to be confined in the $x-y$ plane with the magnetic field $B$ along the $z$-direction which is also the direction of confinement with a typical layer width of $d$ (finite $d$ corresponds to the realistic quasi-2D system studied in the laboratory and $d=0$ corresponds to the strictly 2D idealized system often studied theoretically for convenience), the application of the external magnetic field leads to the Landau quantization of electronic energy levels given by $E_{n}=(n+1 / 2) \hbar \omega_{c}$ where $n=0,1,2,3, \ldots$ is the orbital LL index and $\omega_{c}=e B / m c$ is the cyclotron frequency defining the equidistant energy level separation (i.e., the simple harmonic oscillator spectrum) between the 2D LLs. Each LL has a macroscopic degeneracy given by $\left(2 \pi l^{2}\right)^{-1}$ per unit area where $l=\sqrt{\hbar c / e B} \equiv \sqrt{\hbar / m \omega_{c}}$ is the magnetic length (which is used as the unit of length throughout). For a given 2D electron density of $N_{s}$ (per unit area), one has a LL filling factor $\nu=N_{s} /\left(2 \pi l^{2}\right)^{-1}=2 \pi l^{2} N_{s}$ indicating the filling of the macroscopically degenerate LLs in the 2 D electron system. If $\nu<1$, only the lowest (orbital) Landau level (LLL), by convention denoted as $n=0$, is fractionally occupied by electrons. Our discussion, so far, has neglected electron spin which is equivalent to assuming the 2 D system to be spin polarized (by a sufficiently strong $B$ 
field, for example). Including spin degeneracy in the picture introduces a factor of two since each orbital LL state (i.e., $n=0,1,2, \ldots$ ) can be filled with both up and down spins. Incorporating spin in this "trivial" manner (i.e., assuming each orbital LL to be occupied sequentially by spin up and down electrons), we get that $0<\nu<2$, $2<\nu<4,4<\nu<6$, and so on, correspond, respectively, to spin up-down orbital LLs $n=0$ (LLL), $n=1$ (the second Landau level, SLL), $n=2$ (the third Landau level, TLL). Our goal in this work is to theoretically investigate the FQHE in $n=0,1$, and 2 and provide a critical comparative study of the FQHE in the LLL, SLL, and TLL, emphasizing the key role played by the quasi-2D layer thickness parameter $d$ (or more precisely the dimensionless parameter $d / l$ ) in determining the relative strength, stability, or importance of various FQH states in different orbital LLs. We consider only completely spin-polarized (i.e., spinless) FQH states in our calculations since the primary fractional states $1 / 2,1 / 3$, and $1 / 5$ are universally thought to be spin-polarized.

The motivation of our work stems from the experimental observation that $\mathrm{FQH}$ states are ubiquitous in the LLL (about 70 distinct FQH states have been experimentally observed ${ }^{7,8,9}$ in the $n=0$ LLL with $0<\nu<2$ ), fairly rare in the SLL (less than $10 \mathrm{FQH}$ states have been observed $10,11,12,13,14,15,16$ in the $n=1$ SLL with $2<\nu<4$, and these are much "weaker" than the corresponding LLL FQH states in the sense that the observation of the SLL FQHE requires much lower temperatures and much higher sample mobilities than in the corresponding LL situation), and essentially nonexistent in the TLL (no robust $n=2$, with $4<\nu<6$, TLL FQH state has so far been convincingly observed experimentally 16,17 ). We establish definitively, in this work, that even a qualitative understanding of the higher LL FQHE (i.e., for $n>0$ ) must necessarily include the finite width effect (in the $z$-direction) of the quasi-2D electron layer. We point out that the quantitative role of the layer width in the FQHE (even in the LLL) has long been theoretically known $18,19,20,21,22,23$. What we show in the current work, through the detailed comparison of theoretical numerical results in the $n=0,1,2$ LLs obtained on an equal footing within the same model and approximation scheme, is that the higher (i.e., $n=1,2$ ) LL FQHE has fundamentally different qualitative dependence on the quasi-2D layer width parameter $d / l$ compared with the LLL $(n=0)$ case. In particular, we find that a finite value of $d / l$ is essential in establishing the FQHE in higher LLs whereas in the LLL, finite $d / l$ only serves to quantitatively weaken the FQHE! The FQH states weaken (strengthen) in $n=0$ (1) LLs as $d / l$ increases from the strictly $2 \mathrm{D} d=0$ limit. In the TLL $(n=2)$, we do not find a stable FQHE at all, although our limited numerical results show similar trends in $n=1$ and $n=2$ LLs.

The driving stimulus for studying the FQHE physics in the $n>0$ orbital LLs is, of course, to shed light on the enigmatic $\nu=5 / 2 \mathrm{FQHE}$, originally observed 10 exper- imentally in 1987 and subsequently confirmed and further studied experimentally repeatedly $11,24,25$ over the last two decades. The great fundamental significance of the 5/2 FQHE cannot be overstated. With the obvious exceptions of the original discoveries of the (integer) quantum Hall effect itself $\mathrm{f}^{26}$ and the subsequent $1 / 3 \mathrm{FQHE}^{1}$, the $5 / 2$ FQHE may arguably be the next most important experimental discovery in the field. It is the only known (so far) exception (for a single 2D layer system) to the famous "odd denominator" rule for the FQHE, i.e., the FQHE occurs at an odd denominator filling factor $\nu=p / q$ with $q$ an odd integer (and $p$ either even or odd) with a concomitant quantization of the Hall conductance into a fractionally quantized Hall conductivity $\sigma_{x y}=(p / q)\left(e^{2} / h\right)$ and a zero (or a deep minimum) in the longitudinal conductivity $\sigma_{x x}$ (and also in the longitudinal resistivity $\left.\rho_{x x}=\sigma_{x x}\left[\sigma_{x x}+\sigma_{x y}\right]^{-2}\right)$. All other (i.e., except for the $5 / 2$ state in the SLL) observed FQHE states (e.g., $\nu=1 / 3,2 / 5,3 / 7, \ldots$ in the LLL; $\nu=7 / 3,8 / 3,11 / 5, \ldots$ in the SLL) strictly obey the odd denominator rule, but the $\nu=5 / 2$ state, with its well defined quantized Hall conductance $\sigma_{x y}=(5 / 2)\left(e^{2} / h\right)$, stands in stark contrast to the odd denominator rule. We emphasize that the even denominator nature of the $5 / 2$ FQHE is not only a curious anomaly, it challenges our understanding of the FQHE, as developed ${ }^{2}$ in the Laughlin $\nu=1 / q$ (with $q$ an odd integer) wavefunction and further developed in the Jain composite fermion theory based wavefunctions ${ }^{3.9}$ for the $\nu=p / q$ type FQH states (still with $q$ odd). The odd integer restriction of the filling factor denominator $q$ in the "standard" (i.e., Laughlin-Jain) FQHE model is inescapable since it arises from the Pauli exclusion principle for the electrons. Any even-denominator FQHE must thus fall outside the standard Laughlin-Jain FQHE paradigm, and must somehow correspond to the condensation of bosons (which do not obey Pauli principle and therefore allow for even denominator FQHE) in the $\nu=5 / 2$ incompressible FQH liquid.

As an aside, it is worthwhile to mention that the $5 / 2$ state is the only observed even-denominator FQHE, as emphasized above, only for single-layer 2D systems. In bilayer (or more generally, multilayer) 2D systems, where experiments are carried out in two parallel 2D layers separated by a barrier (i.e., a double quantum well structure), even-denominator (e.g., $\nu=1 / 2,1 / 4) \mathrm{FQH}$ states have been observed $27,28,29,30$ rather routinely. These bilayer FQH states are theoretically well-understood 31 to be strong-coupling paired Laughlin states, which were postulated by Halperin ${ }^{32}$ some time ago. For example, the observed $\nu=1 / 2$ bilayer $\mathrm{FQH}$ state $\frac{27,28}{28}$ has been shown 31 to be the Halperin 331 state ${ }^{32}$, where tightly bound pairs of electrons condense into a bosonic Laughlin state which is allowed to describe even-denominator fractions since the Pauli principle does not apply to bosons. Such strongly paired Halperin even-denominator states are Abelian states, similar to ordinary Laughlin FQH states, in contrast to the Moore-Read non-Abelian Pfaffian even-denominator state ${ }^{33}$ (see below) which is 
thought to describe the weakly paired BCS state underlying the $5 / 2$ single-layer even-denominator $\mathrm{FQH}$ state. We also mention here that no single-layer (as opposed to bilayer) $\nu=1 / 2$ FQHE has even been observed experimentally although we know of no fundamental reason ruling out such paired (either strong-coupling or weakcoupling) LLL states.

The leading theoretical candidate for the $\nu=5 / 2$ (and its electron-hole counterpart at $\nu=7 / 2$ ) FQH state is the so-called "Pfaffian" wavefunction 33 of Moore and Read (MR), which has been extensively studied over the last 17 years $34,35,36,37,38,39,40,41,42,43,44,45$. In fact, there is no other proposed viable candidate ground state wavefunction for the observed 5/2 FQH state, and as such, a consensus has emerged that the Moore-Read Pfaffian wavefunction is the likely description for the enigmatic evendenominator 5/2 FQHE. In spite of this consensus, arising primarily out of a lack of any other viable alternative, there has been a minority viewpoint ${ }^{40,41}$ questioning the validity of the MR Pfaffian in describing the $5 / 2 \mathrm{FQH}$ ground state. In addition, a nagging issue of substantial importance is why there is an incompressible FQH state at half filling in the SLL (i.e., $\nu=2+1 / 2=5 / 2$ ), but not at $\nu=1 / 2$ in the LLL (or for that matter, at half filling in the TLL, i.e. $\nu=4+1 / 2=9 / 2)$ since the Pfaffian wavefunction carries no LL index label and is presumably an allowed variational description for the half-filled LL in any orbital level $n=0,1,2, \ldots$ Important early work ${ }^{34,35}$ showed that the Pfaffian wavefunction is a rather fragile description of the $\nu=5 / 2 \mathrm{FQH}$ state, and slight variations in the effective interaction between the carriers, as, for example, could arise from the finite layer width or from changing the orbital LL index could, in principle, affect the validity of the Pfaffian wavefunction as a suitable description of the $\nu=5 / 2$ ground state. The "mundane" details of the dependence on the effective electron-electron interaction, rather than any deep fundamental principle, is, in fact, the reason for the SLL 5/2 state to be an incompressible FQHE state whereas the corresponding LLL $1 / 2$ state is a compressible Fermi liquid state. Thus, the orbital LL and the finite layer thickness effects (and also perhaps the interLandau level coupling, an effect we uncritically ignore in this work) on the effective electron-electron interaction are the key in determining the relative stability of various incompressible FQH states in different orbital LLs.

In this work, we concentrate on three primary FQH states at filling factors $1 / 2,1 / 3$, and $1 / 5$ in three orbital LLs $n=0,1,2$, and theoretically investigate the relative stability of the incompressible quantized Hall states at these fillings by calculating, as a function of the finite width parameter, the ground state wavefunction overlap between the exact (numerically exactly diagonalized) few-particle ground state wavefunction with the corresponding candidate variational wavefunction (i.e., Laughlin for $\nu=1 / 3,1 / 5$ and MR Pfaffian for $\nu=1 / 2$ ) for the incompressible FQH state. A high (low) overlap provides a strong hint that the corresponding realistic
FQHE state is (is not) described by the corresponding variational state (i.e., Laughlin for 1/3, 1/5 and MooreRead for $1 / 2$ ) in the appropriate orbital LL. Such exact diagonalization studies of small systems have been the main theoretical tool in learning about the nature of incompressible FQH states ever since the original discovery of the FQHE. In particular, the universal acceptance of the celebrated Laughlin wavefunction as the appropriate description for the observed $1 / 3 \mathrm{FQH}$ state in the LLL is based almost entirely on the remarkably large (essentially unity) overlap between the analytical Laughlin wavefunction and the exact small-system numerical wavefunction. Similarly, the Jain composite fermion theory based variational wavefunctions are thought to be excellent descriptions for the non-primary (i.e., $\nu=m /(2 p m \pm 1)$-with $m>1$ and $p$ an integer-such as $\nu=2 / 5,3 / 7,4 / 9$, etc.) LLL FQH states because of the good overlap between the composite fermion wavefunctions and exact numerical wavefunctions for small systems. Even the MR Pfaffian wavefunction is accepted to be the reasonable description for the experimental SLL $1 / 2$ state (i.e., at $\nu=2+1 / 2=5 / 2$ ) based simply on the observation, made originally by Morf ${ }^{34}$ and followed up in other subsequent theoretical publications 35,39 , that the Pfaffian has good, albeit not spectacular, overlap $(\sim 0.8-0.9$ for the strict $2 \mathrm{D}$ system) with the exact small-system numerical wavefunction. Conversely, alternative possibilities for the 5/2 FQHE state, such as the Halperin 331 state 32 or the Haldane-Rezayi spin-singlet hollow-core $\underline{46}$ state, are ruled out 29,30 theoretically essentially entirely on the basis of very poor calculated overlap of these candidate states with exact small system numerical wavefunctions.

Another measure of the stability-besides the wavefunction overlap-that applies to the MR Pfaffian state, in particular, is the appearance of the threefold topological degeneracy in the torus geometry that is a signature of the Pfaffian state. This degeneracy is a direct signature of the non-Abelian nature of the state (and therefore would not arise if the state is Abelian such as the Halperin 331 state). The existence of this degeneracy for a physical interaction, i.e., a modified Coulomb interaction, has not been earlier reported in the literature. Our finding of the appropriate MR degeneracy in the $5 / 2$ numerical state precisely where the overlap is maximal is an important advance in theoretical understanding.

We choose the Laughlin (for $1 / 3$ and $1 / 5$ ) and the Pfaffian (for $1 / 2$ ) as our candidate variational ansatz because these are the only proposed incompressible FQH states at these primary fractional fillings. Our work will, therefore, miss out on discovering any other possible ground state wavefunctions (i.e., other than Laughlin for $1 / 3$ and $1 / 5$ and Moore-Read for 1/2) describing the experimental FQH state since we restrict our overlap calculations entirely to the Laughlin or Moore-Read candidate states. This restriction is, however, not a serious drawback of our work since no other candidate wavefunctions exist in the literature for the FQH states of our interest.

Another incentive for our work comes from the fact 
that existing numerical work ${ }^{47,48,49,50}$, much of it carried out in the idealized $2 \mathrm{D}$ limit, concludes that the $1 / 3$ FQH state in the SLL (i.e., the $\nu=2+1 / 3=7 / 3$ state or its electron-hole counterpart $\nu=8 / 3$ state) is unlikely to be a simple Laughlin state (although in Ref. 48 the deviation from the Laughlin wavefunction stems from residual quasiparticle interactions) since the SLL smallsystem exact diagonalization studies give only modest wavefunction overlap between the Laughlin wavefunction and the exact numerical ground state in the SLL. As mentioned above, for the $5 / 2 \mathrm{FQH}$ state, the calculated overlap 34,35,39 between the MR Pfaffian and the exact diagonalization finite system wavefunction is also quite modest, much below unity, in the idealized 2D limit. Our results would shed light on whether the finite-thickness, or width, of the quasi-2D layer enhances the overlap between the Laughlin (or Moore-Read) state and the exact wavefunction so that the Landau level dependent stability of the incompressible FQHE can be discussed in terms of a systematic tuning of the Hamiltonian through the variation in the electron-electron interaction caused by changing the layer width parameter $d / l$. We find that indeed a finite $2 \mathrm{D}$ layer width, i.e., a true quasi-2D system, is necessary for stabilizing the FQHE in the SLL, at least within the restricted wavefunction space (i.e., Laughlin and Moore-Read) we investigate. We note that increasing $d / l$ from zero (i.e., the strict $2 \mathrm{D}$ ideal limit) is equivalent to softening the interaction.

One rather direct consequence of the quasi-2D finitethickness effect on the FQHE is the orbital coupling of an in-plane (i.e., parallel to the 2D layer) applied magnetic field, in addition to the quantizing perpendicular magnetic field. Having both parallel and perpendicular magnetic fields is, of course, equivalent to having a tilted magnetic field, which is often used in $2 \mathrm{D}$ physics to study spin polarization effects - the idea being that the parallel component of the magnetic field couples only to the electron spin through the modification of the Zeeman energy. This is certainly true in the idealized strictly $2 \mathrm{D}$ limit where a magnetic field in the $2 \mathrm{D}$ plane has no coupling to the orbital $2 \mathrm{D}$ dynamics of the electrons, but does couple to the electron spin. In a quasi-2D system, however, the situation is more complex since an in-plane field could, in principle, couple to the quasi-2D orbital dynamics of the electrons through the finite-thickness of the electron layer. One effect of the parallel field would, for example, be to squeeze the electron layer in the third direction, reducing its effective quasi-2D layer width. We consider such magneto-orbital coupling effects due to a parallel applied field in our work within the context of our LL-dependent finite-thickness studies, using a parabolic or simple harmonic confinement model.

A compelling and timely reason for the detailed investigation of higher LL FQHE is the recent interest in using the $\nu=5 / 2$ (and possibly $\nu=12 / 5$, recently observed by Xia, et al in Ref. 13 , and $\nu=13 / 5$ ) FQH states for fault-tolerant topological quantum computation 51,52 using the non-Abelian quasiparticles associated with the
Pfaffian state. Since the non-Abelian nature of the quasiparticle excitations is crucially tied to the specific form of the MR Pfaffian wavefunction, it is important that we know whether the observed 5/2 state is really the MooreRead state or not. Recently, serious questions have been raised $^{40,41}$ about whether the Pfaffian is the appropriate description for the $5 / 2$ state. Our work, investigating the detailed nature of the incompressibility as a function of the quasi-2D layer width parameter in higher LLs, thus is timely and necessary for further progress in the subject of topological quantum computation. There have also been several recent experimental investigations of the SLL FQHE motivated by topological quantum computation considerations $53,54,55$.

The rest of the paper is organized as follows: In section IIA we introduce the essential Hamiltonians we are studying, namely, the electron-electron interaction Hamiltonian and ones that give either the Laughlin or Pfaffian states as zero-energy ground states. In section II B we describe the various models used to characterize the quasi-2D nature of the experimental quantum confinement, i.e. the "finite-thickness" models for various physical systems. The $f$ - and $g$-functions (defined below) are then considered in section $\amalg \mathrm{C}$ as a first attempt to understand the effective electron-electron interaction due to the quasi-2D nature of the system. Overlaps between the exactly diagonalized system for the "realistic" quasi$2 \mathrm{D}$ system and the Laughlin (fillings $1 / 3$ and $1 / 5$ ) or Pfaffian (filling 1/2) wavefunction as a function of the quasi-2D layer width are reported in section III A The appearance of the ground state threefold degeneracy signature of the MR Pfaffian state on the torus-particularly for quasi-2D systems-is investigated in Sec. IIIB. The effect of an in-plane magnetic field and the quasi-2D nature of the system are considered in section IIIC In section [V we discuss connections of our overlap results to previous work and, in particular, whether or not the physics of the FQHE is adequately captured by the first few pseudopotentials. The finite thickness effects on the excitation gaps are also briefly discussed in section $\mathrm{V}$. Finally, conclusions are given in section VI with some additional discussions. A short letter reporting some of our results has recently appeared in the literature ${ }^{56}$.

\section{MODEL}

\section{A. Hamiltonians: Coulomb, Laughlin, and Pfaffian}

We consider spin polarized electrons entirely confined (i.e., no inter-LL coupling) to a Landau level of index $n=0$ (LLL), 1 (SLL), and 2 (TLL) interacting through a pair potential $V\left(r_{i j}\right)$, where $r_{i j}=\left|\vec{r}_{i}-\vec{r}_{j}\right|$ is the distance between two electrons (distance is measured in units of magnetic length $l$ ). Since the electrons are confined to a single LL the kinetic energy is a constant, therefore, the Hamiltonian for $N$ electrons is taken to be the interaction 


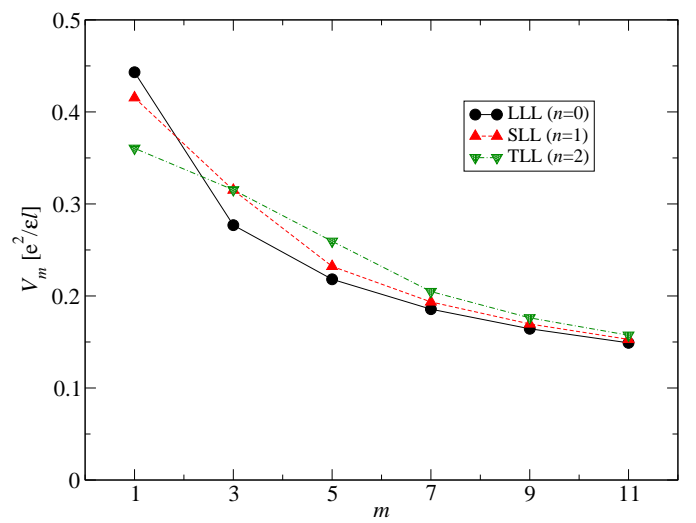

FIG. 1: (Color online) $V_{m}$ as a function of $m$ ( $m$ odd) for the pure Coulomb potential (zero-thickness), i.e., $V(k)=$ $\left(e^{2} l / \epsilon\right)(1 / k)$, in the LLL (solid circle), the SLL (upward triangle), and the TLL (downward triangle). $V_{m}$ are given in units of $e^{2} / \epsilon l$. The lines are a guide to the eye.

Hamiltonian

$$
\hat{H}=\sum_{i<j}^{N} V\left(r_{i j}\right) .
$$

Haldane $e^{57,58}$ showed how this Hamiltonian can be parameterized by the relative angular momentum $m$ between two electrons, through an expansion in the "pseudopotential" functions $V_{m}^{(n)}$, which serve as a complete set of basis functions due to angular momentum conservation:

$$
\hat{H}=\sum_{i<j}^{N} V\left(r_{i j}\right)=\sum_{m=1(\text { odd })}^{\infty} V_{m}^{(n)} \sum_{i<j}^{N} \hat{P}_{m}\left(m_{i j}\right)
$$

where $\hat{P}_{m}\left(m_{i j}\right)$ is an operator that projects onto the states of relative angular momentum $m_{i j}=m$. Since we are considering spin polarized fermions only odd pseudopotentials are relevant. The Haldane pseudopotentials $V_{m}^{(n)}$ for electrons confined to a LL with index $n$, in the planar geometry (as opposed to the spherical geometry), are written as

$$
V_{m}^{(n)}=\int_{0}^{\infty} d k k\left[L_{n}\left(k^{2} / 2\right)\right]^{2} L_{m}\left(k^{2}\right) e^{-k^{2}} V(k)
$$

where $L_{n}(x)$ are Laguerre polynomials, and $V(k)$ is the Fourier transform of the interaction potential $V(r)$. To define our Fourier transform convention we write

$$
\begin{aligned}
V(k) & =\frac{1}{2 \pi} \int d^{2} k e^{i \vec{k} \cdot \vec{r}} V(r) \\
& =\int_{0}^{\infty} d r r J_{0}(k r) V(r) .
\end{aligned}
$$

This parameterization allows all the calculations to be done entirely within the Hilbert space of the lowest Lan- dau level, i.e., the information about higher LLs is completely contained within the $V_{m}^{(n)}$. Note that this simplification depends on our neglecting all Landau level mixing effects, which, along with the assumption of complete spin-polarization, is a key and uncritical assumption for our theory.

In a purely $2 \mathrm{D}$ system the electron-electron interaction is the Coulomb interaction $V(r)=\left(e^{2} / \epsilon l\right)(1 / r)$, where $r$ is the distance in the 2D plane between a pair of electrons, yielding a Fourier transform of $V(k)=\left(e^{2} l / \epsilon\right)(1 / k)$ where $k$ is units of $1 / l$. Figure 1 displays the first six pseudopotentials as a function of $m$ for the LLL, the SLL, and the TLL. Although the differences between the pseudopotentials in different LLs are small quantitatively, the qualitative changes in the system behavior can be severe, as discussed below. The main qualitative difference in the pseudopotentials $V_{m}^{(n)}$ among the three LLs is that $V_{1}^{(0)}>V_{1}^{(1)}>V_{1}^{(2)}$, but $V_{2}^{(0)}<V_{2}^{(1)}, V_{2}^{(2)}$ and $V_{3}^{(0)}<V_{3}^{(1)}<V_{3}^{(2)}$.

We will denote the celebrated Laughlin 2 wavefunction at $\nu=1 / q$ to be $\left|\Psi_{L}\right\rangle$. It was shown by Haldane 57 that $\left|\Psi_{L}\right\rangle$ at filling $\nu=1 / 3$ is the exact zero-energy ground state for a "hard-core" Hamiltonian $\hat{H}_{L}^{(3)}$ where

$$
\hat{H}_{L}^{(3)}=(\text { const. }) \sum_{i<j}^{N} \hat{P}_{1}\left(m_{i j}\right) .
$$

This can be obtained from the original Hamiltonian (Eq. 2) by setting $V_{1}^{(n)}=$ constant and $V_{m}^{(n)}=0$ for all $m \geq 3$; in other words, the Laughlin state at $1 / 3$ avoids all electron pairs with $m<3$ since $V_{1} / V_{m}=\infty$ for $m \geq 3$. Further, the Laughlin state at $\nu=1 / q$ (filling $1 / 5$ for example) is the exact zero-energy ground state for a Hamiltonian

$$
\hat{H}_{L}^{(q)}=\text { (const.) } \sum_{m=1 \text { (odd) }}^{q-2} \sum_{i<j}^{N} \hat{P}_{m}\left(m_{i j}\right) .
$$

Generally, $\hat{H}_{L}^{(q)}$ is a Hamiltonian that penalizes two electrons that have an angular momentum smaller than $m$.

The Pfaffian ${ }^{33}$ wavefunction $\left|\Psi_{P f}\right\rangle$ is thought to be the leading candidate for the FQHE at filling $1 / 2$ in the SLL, i.e., $\nu=5 / 2$, and is known ${ }^{59}$ to be an exact zero-energy ground state for a three-body Hamiltonian $\hat{H}_{P f}$ which penalizes states where three electrons are in a relative angular momentum state smaller than some value. There is no known two-body interaction Hamiltonian which has the Pfaffian state as the ground eigenstate, so we should think of the Pfaffian as a variational ansatz for our twobody interaction Hamiltonians.

(We are providing necessarily very brief discussions of the Laughlin and Pfaffian Hamiltonians in order to facilitate easier discussions in later sections of this work.)

In the pure 2D system, where the above discussion applies, the electron interaction is just pure Coulomb, however, the finite extent of the single-particle electron 


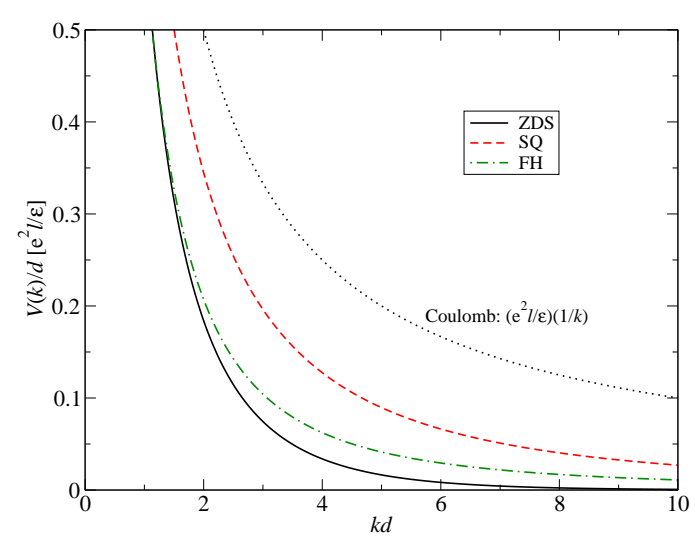

FIG. 2: (Color online) Fourier transform of the finitethickness potentials divided by thickness $d$, i.e., $V(k) / d$ versus $k d$ for the ZDS (solid line), SQ (dashed line), and FH (dashed-dotted line) potentials. Also shown is the Coulomb potential.

wavefunction in the direction perpendicular to the plane in actual experimental quasi-2D systems will modify the electron interaction as discussed below.

\section{B. Finite-thickness Modeling Potentials}

Depending on the details of the physical systems (i.e., quantum wells, heterostructures, etc.), there are many possible models for the inclusion of the finite-thickness effect with the 2D Coulomb interaction. We do not, however, anticipate much qualitative difference among these different models since they all lead to the softening of the Coulomb potential, with the softening depending crucially on the largeness of the thickness parameter $d / l$. In particular, the pseudopotentials change in the presence of the finite-thickness since, in addition to the magnetic length, a new length scale $d$ characterizing the quasi-2D thickness becomes relevant, and for $d \gg l$, the pseudopotentials are modified substantially from their $d=0$ ideal $2 \mathrm{D}$ Coulomb values.

We consider the three most extensively used finitethickness potential models, for which we give the Fourier transforms, that seek to model the effect of the quasi2D nature of the experimental system, namely, (1) the Zhang-Das Sarma (ZDS) potential20, which was introduced specifically to theoretically model finite thickness effects on the FQHE,

$$
V_{Z D S}(k)=\frac{e^{2} l}{\epsilon} \frac{e^{-d k / 2}}{k},
$$

(2) the infinite square-well (SQ) potentia ${ }^{60}$, which is appropriate for 2D GaAs quantum well structures,

$$
V_{S Q}(k)=\frac{e^{2} l}{\epsilon} \frac{1}{k} \frac{\left\{3 k d+\frac{8 \pi^{2}}{k d}-\frac{32 \pi^{4}\left(1-e^{-k d}\right)}{(k d)^{2}\left[(k d)^{2}+4 \pi^{2}\right]}\right\}}{(k d)^{2}+4 \pi^{2}},
$$

and (3) the Fang-Howard (FH) variational potential ${ }^{61,62}$ for a heterostructure

$$
V_{F H}(k)=\frac{e^{2} l}{\epsilon} \frac{9}{8 k} \frac{\left(24+9 k d+(k d)^{2}\right)}{(3+k d)^{3}} .
$$

Potentials (2) and (3) are found by using single-particle electron wavefunctions in the $z$-direction of $\eta(z)=$ $\sqrt{2 / d} \cos (\pi z / d)$ and $\eta(z)=\sqrt{27 / 2 d^{3}} z \exp (-3 z / 2 d)$, respectively (these functions are given merely to make our definitions of the thickness parameter $d$, for each model, completely clear). In the above, for (1) and (2), $d$ is the width of the electron layer (in units of $l$ ) in the $z$ direction, and for (3) it parameterizes the layer thickness variationally. Obviously, as $d \rightarrow 0$, all of the above finitethickness potentials, describing quasi-2D systems, reduce to the pure 2D Coulomb potential $V(k)=\left(e^{2} l / \epsilon\right)(1 / k)$. Fig. 2 shows the Fourier transforms divided by $d$ as functions of $k d$ for each finite-thickness potential used clearly indicating the "softening" of the Coulomb potential. Note that we keep the background lattice dielectric constant $\epsilon$ in our definition of the 2D Coulomb interaction only for the sake of completeness with $e^{2} /(\epsilon l)$ being our energy unit (and ' $l$ ' the length unit). We note that our definition of $d$ as the relevant thickness or width parameter for each model of quasi-2D confinement is contained entirely in Eqs. [7.9-this is important since later we introduce alternative width parameters $d^{\prime}$ and $w$.

As previously mentioned, the purpose of this work is not to determine the quantitative accuracy of some particular finite-thickness model compared to experimental systems. Rather, we are interested in the possible nontrivial qualitative changes that can occur when considering realistic potentials which are not pure $2 \mathrm{D}$ Coulomb. We note, however, that the SQ and FH models correspond to the two most common quasi-2D experimental systems (quantum well and heterostructure, respectively) whereas the ZDS model, while not corresponding to any physical system, is extensively used in FQH theoretical studies.

For the sake of completeness we provide the first six pseudopotentials for all three finite-thickness potentials as functions of $d$ in the LLL, the SLL, and the TLL shown in the left, middle, and right panels of Fig. 3 In all LLs shown, the finite-thickness has the effect of reducing (or softening) all of the pseudopotentials in a rather "trivial" way, that is, there is no crossing or non-monotonic behavior: $V_{1}^{(n)}>V_{3}^{(n)}>V_{5}^{(n)}>\ldots$ remains for all $d$. It is clear, however, that for the SQ and FH potentials the softening as $d$ increases is less severe compared to the ZDS potential. (We mention as a cautionary note that although the same thickness parameter $d$ has been used in Figs. 2 and 3 for our three quasi-2D models, the parameter has somewhat different meaning in the three cases as can been seen from their strongly different quantitative effects on the pseudopotential softening in the three models as is obvious from Figs. 7],8,) Another qualitative feature to note is that it is visually difficult to notice any 


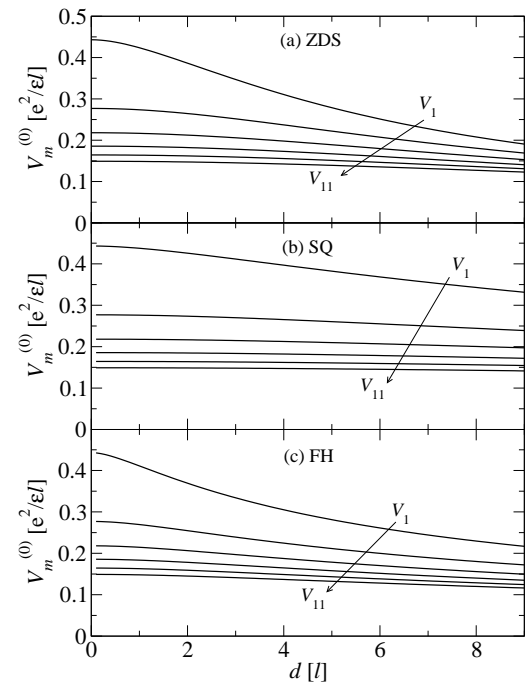

LLL

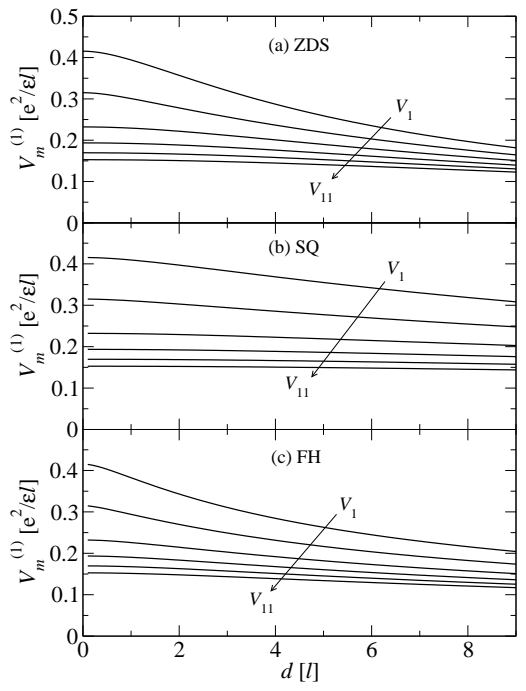

SLL

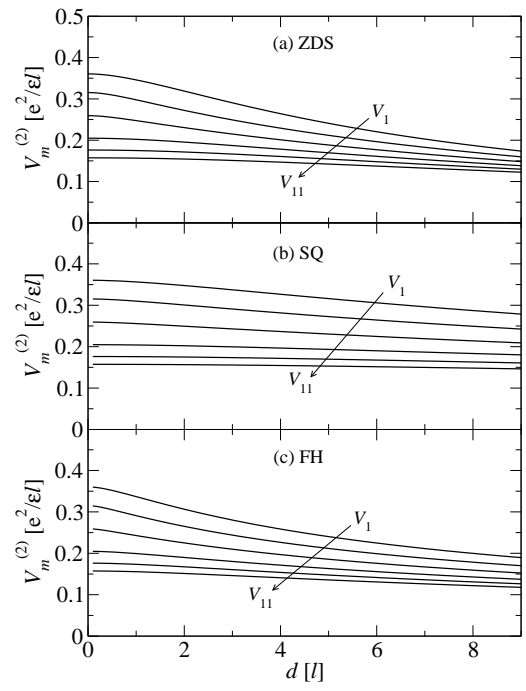

TLL

FIG. 3: Pseudopotentials for the LLL (left), the SLL (middle), and the TLL (right). Each panel has three plots each displaying the $V_{m}$ 's for the three different finite-thickness modeling potentials, which are labeled: (a) Zhang-Das Sarma (ZDS), (b) infinite square-well (SQ), and (c) Fang-Howard heterostructure (FH). The units of the $V_{m}$ 's are as in Fig. 1 .

striking difference between the behavior of the pseudopotentials, themselves, in different orbital LLs.

We have actually carried out calculations for a fourth model, the parabolic quantum well (PQW) (or the Gaussian confinement model), which we discuss in Sec. IIIC

\section{C. $f$ - and $g$-functions}

Only the relative differences in the pseudopotentials are important in characterizing the physical nature of the FQH ground state. Following Ref. 19 we form the dimensionless $f$-functions defined through

$$
f_{m}^{(n)}=\frac{V_{3}^{(n)}-V_{m}^{(n)}}{V_{1}^{(n)}-V_{3}^{(n)}}
$$

which quantitatively describe how close a given Hamiltonian is to $\hat{H}_{L}^{(3)}$ that produces the $1 / 3$ Laughlin state as the exact ground state.

From the definition, it is clear that $f_{1}^{(n)}=-1$ and $f_{3}^{(n)}=0$. As described in Ref. 19 the $f$-function is useful because for $\hat{H}_{L}^{(3)}$ all $f_{m}^{(n)}=0$ for $m \geq 3$, and hence, any interaction that produces $f$-functions with this property will be exactly described by the Laughlin state for $\nu=1 / 3$. Further, if the $f_{m}^{(n)}$ are very small for $m>3$ then the exact state will be well approximated by the Laughlin state for $\nu=1 / 3$, so, by the simple computation of the $f$-functions for some Hamiltonian (or interaction potential) one can get an idea of how well the Laughlin state will describe the actual ground state. Thus, the $f$ functions far better manifest Laughlin wavefunction-like correlations than the pseudopotentials (the $V_{m}^{(n)}$ functions) themselves.

One can generalize this to functions which characterize how close a given Hamiltonian is to $\hat{H}_{L}^{(q)}$ that produces the $\nu=1 / q$ Laughlin state as the exact ground state. To that end we define $g$-functions to handle the $\nu=1 / 5$ Laughlin state as

$$
g_{m}^{(n)}=\frac{V_{5}^{(n)}-V_{m}^{(n)}}{V_{1}^{(n)}-V_{5}^{(n)}} .
$$

The $g$-functions satisfy $g_{1}^{(n)}=-1$ and $g_{5}^{(n)}=0$. The "hard-core" aspect of a potential is displayed by $g_{m}^{(n)}=0$ for all $m \geq 5$. One could go further with this procedure defining $h$-functions for investigating the "Laughlin-ness" of a Hamiltonian at $\nu=1 / 7, i$-functions for $\nu=1 / 9$, etc.

Figure 4 shows the $f$-functions as a function of thickness $d$ for the finite-thickness potentials we have considered, namely, the ZDS (top labeled (a)), SQ (middle labeled (b)), and FH (bottom labeled (c)) for the $n=0$ LLL (left panel), the $n=1$ SLL (middle panel), and the $n=2$ TLL (right panel). Note that the $f$-functions for the ZDS potential in the LLL were shown previously in Ref. 19 as well as the $f$-functions for the $\mathrm{FH}$ potential in the SLL in Ref. 63. For the LLL (left panel of Fig. (4) we observe that for zero-thickness $d=0$ all $f_{m}^{(0)}<1$ and it is known (and shown below with other measures) that the Laughlin state for $1 / 3$ is a very good approximation to the exact state. However, as $d$ is increased all $f$ 's that are free to vary $\left(f_{3}^{(0)}\right.$ through $\left.f_{11}^{(0)}\right)$ become larger monotonically. It is interesting to note that for the SQ and $\mathrm{FH}$ potentials there is very little increase in the $f$ 's for 


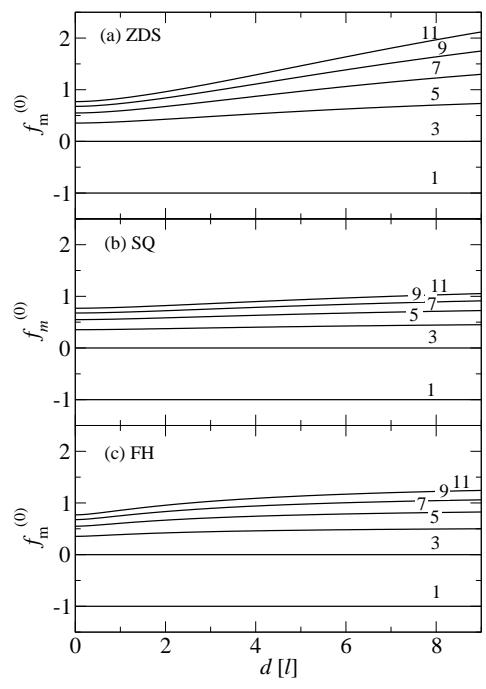

LLL

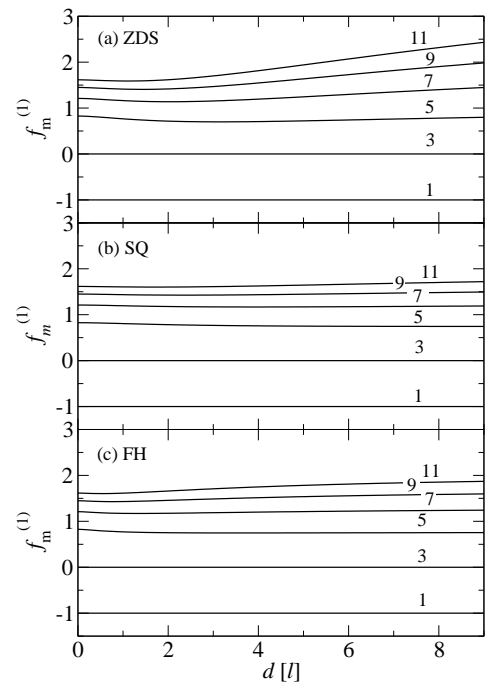

SLL

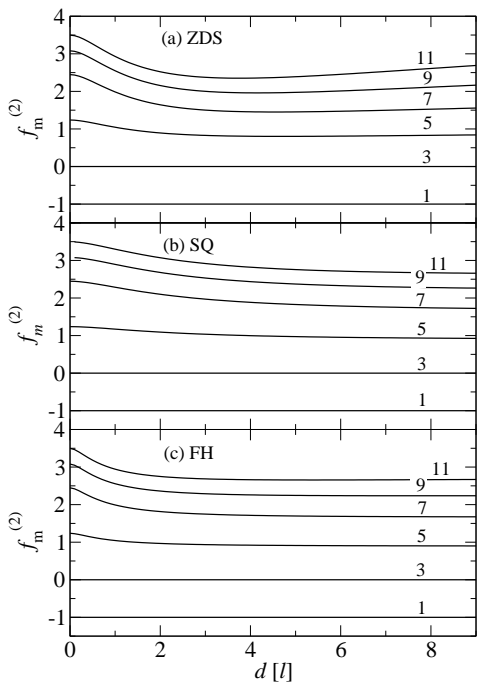

TLL

FIG. 4: $f$-functions for the LLL (left), the SLL (middle), and the TLL (right). Each panel has three plots each displaying the $f$-functions for the three different finite-thickness modeling potentials which are labeled: (a) Zhang-Das Sarma (ZDS), (b) infinite square-well (SQ), and (c) Fang-Howard heterostructure (FH). The $f$-functions, by construction, are dimensionless.

increasing $d$ compared to the ZDS potential, i.e., ZDS overestimates the finite-thickness effect compared with the SQ and FH models. This is also apparent in Figs. 2 and 3 .

From the calculation of the $f_{m}^{(0)}$ 's, as in Ref. 19, one can determine that for some finite-thickness modeling potentials (e.g., ZDS) the Laughlin state is not a good description of the physics for $d$ beyond some value whereas for other potentials (SQ and $\mathrm{FH}$ ) the Laughlin state most likely remains a good description of the physics for all $d$, but the description becomes progressively poorer with increasing $d$. (This suggestion is further investigated below by calculating the overlap between the Laughlin state and the exact ground state as a function of $d$.)

We now investigate $f$-functions in the SLL: The middle panel of Fig. 4 shows $f_{m}^{(1)}$ for all potentials considered. These functions are very similar to those of the LLL, at least qualitatively; they increase essentially monotonically as a function of $d$, however, there is a difference. Specifically, for $d=0$, only $f_{5}^{(1)}$ is below unity while all $f_{7}^{(1)}$ through $f_{11}^{(1)}$ are between 1 and 2 . This already indicates that perhaps the Laughlin state will not be a very good description of the physics here, and, in fact, this has been known for some time $\underline{\underline{49}}$.

However, there is another property the $f$-functions reveal as $d$ is increased. The $f$-functions for the SQ potential were previously calculated in Ref. 63 and it was remarked that in the SLL they do not increase monotonically the way they do in the LLL. Instead there is a weak minimum for intermediate $d$. That being said, the minimum for the SQ potential is weak and $f_{7}^{(1)}$ through $f_{11}^{(1)}$ are still greater than unity for all $d$. Hence, while the
Laughlin state does become a better description for finite $d$ compared to $d=0$ it still never becomes essentially exact, as in the LLL. In our calculations we observe this sort of behavior for the ZDS and FH potentials: an initial decrease in the $f$-functions before a monotonic increase.

Finally, the right panel of Fig. 4 displays the $f$ functions for the TLL where the behavior follows the trend seen from the LLL to the SLL. Namely, $f_{5}^{(2)}$ through $f_{11}^{(2)}$ are all greater than unity for $d=0$ with $f_{11}^{(2)} \sim 3.5$. Clearly, the Laughlin state will not be a good description of the physics for $d=0$ in the TLL. However, as $d$ increases the minimum is much more marked and, in fact, for SQ and $\mathrm{FH}$ potentials at $d=0 f$-functions are maximum.

We now consider the $g$-functions in order to investigate the physics at filling factor $1 / 5$. We would expect the Laughlin $1 / 5$ state to accurately represent the physics if $g_{m}^{(n)} \sim 0$ for $m \geq 5$. The left and middle panels of Fig. 5 display the $g$-functions for the LLL and SLL, respectively, for the three model potentials. It is clear that for $d=0$ in the LLL and SLL the Laughlin state is likely a good representation of the exact state. This is because $g_{m}^{(0)}$ and $g_{m}^{(1)}$ are less than 0.5 for $m \geq 5$. On the other hand, in the TLL (the right panel of Fig. 5) we observe that, for $d=0,1>g_{m}^{(2)}>0.5$, indicating that perhaps the $1 / 5$ Laughlin state is not a good physical description of the exact $\nu=1 / 5$ state in the TLL.

As a function of the thickness parameter $d$ the $g$ functions behave in much the same way as the $f$ functions, that is, for the ZDS, SQ, and FH potentials they increase monotonically as a function of $d$. The main difference between the two is that the local minimum in 


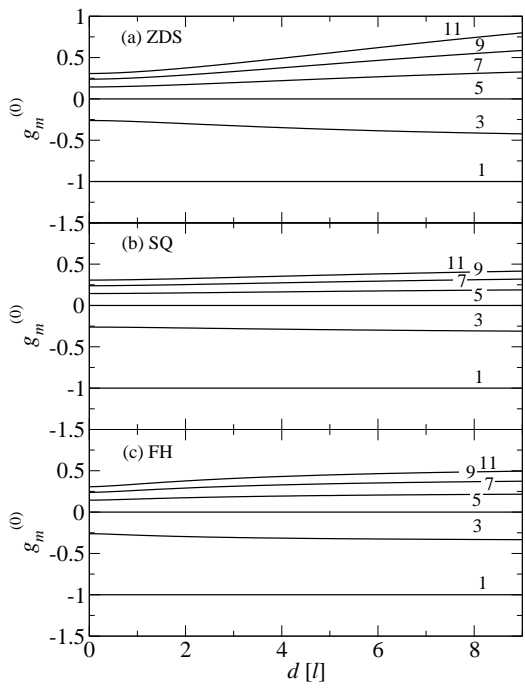

LLL

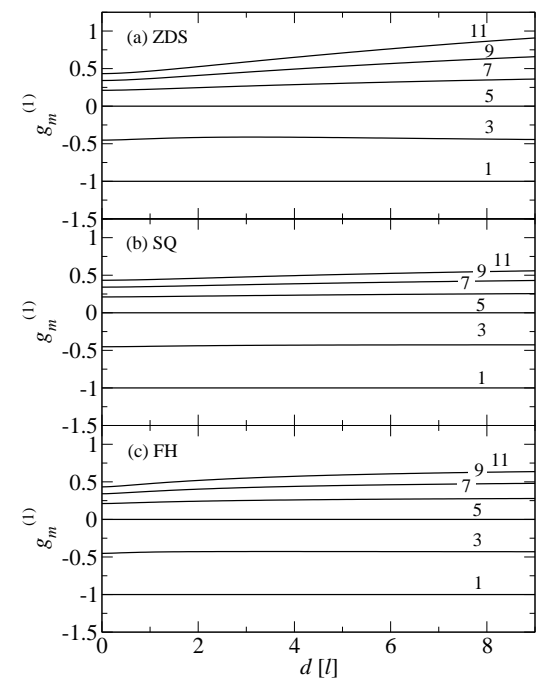

SLL

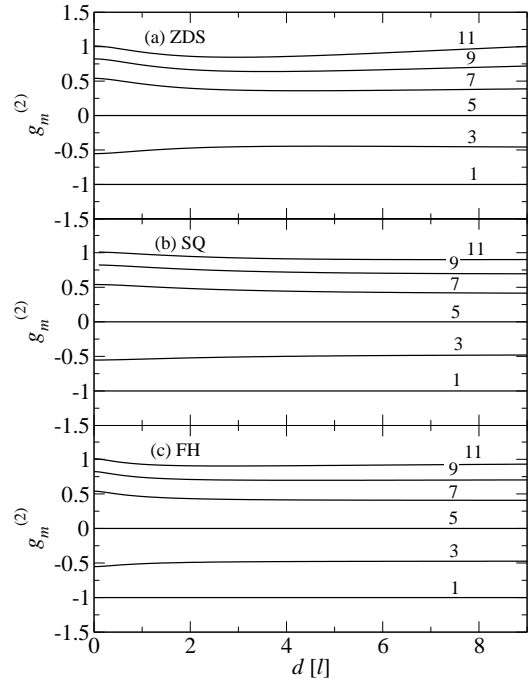

TLL

FIG. 5: As in Fig. 4 except for $g$-functions.

the $g$-function is obtained for finite $d$ only in the TLL as opposed to the SLL for the $f$-function (cf. Fig. 4). A global minimum is obtained in $g$ for the TLL (right panel) for the ZDS potential for finite $d$ while for the SQ and $\mathrm{FH}$ potentials the minima are obtained for very large $d$.

It is clear from inspection that one can obtain a rough qualitative idea of how well the Laughlin state at $1 / 5$ will represent the physics as a function of $d$, namely, in the LLL the 1/5 Laughlin state will be a good description for up to quite large $d$ for the ZDS potential before presumably losing out to some other state. The SQ and FH potentials, on the other hand, will mostly likely produce a state that is very similar to the $1 / 5$ Laughlin for all $d$. A similar prediction is made for the SLL.

For the TLL, however, the ZDS potential will produce a state that is likely not Laughlin-like for small $d$, a state better described by Laughlin for intermediate $d$, and becomes unlike Laughlin again for large $d$. The SQ and FH potentials are likely to produce a state that is consistently unlike Laughlin for all $d$.

Our qualitative discussion based on the thicknessdependent behavior of $f$ - and $g$-functions is consistent with experimental findings: The Laughlin FQH state is abundant in the LLL, scarce in the SLL, and essentially non-existent in the TLL. We emphasize that, by contrast, no such qualitative discussion is possible with respect to the relative abundance of the Moore-Read even-denominator state in various LLs since the MR state, unlike the Laughlin state, is not an exact ground state of any known two-body Hamiltonian, and therefore pseudopotential-based functions such as $f$ and $g$ do not provide any direct insight into the MR state.

The $f$ - and $g$-functions provide a guide to our intuition and, perhaps, a qualitative understanding. How- ever, a way to quantitatively understand the quality of the physical description of the Laughlin state is provided by calculating overlaps with exact wavefunctions which we discuss in the next section.

\section{RESULTS}

\section{A. Overlaps}

A measure of how accurate a variational wavefunction (Laughlin or Pfaffian) is compared to the exact wavefunction is encapsulated in the calculation of the overlap between the two wavefunctions. An overlap of unity means the variational state is exact and a vanishing overlap means the variational state is completely unlike the exact state, perhaps due to different symmetries (different total angular momentum for example). Although the use of overlap calculations in establishing the nature of the incompressible FQH states has been a central conceptual and theoretical tool in FQHE studies, it should be emphasized that the overlap calculation has its limitation since it can only make statements about (necessarily small) finite systems and specific FQH ansatz wavefunctions (e.g., Laughlin, Pfaffian). In spite of these limitations the calculation of wavefunction overlap between exact numerical wavefunctions for small systems with ansatz variational wavefunctions has been a standard theoretical FQHE tool for almost 25 years.

Before we calculate overlaps we make a technical point. To calculate properties of the states and diagonalize the Hamiltonians we have made use of the spherical geometry ${ }^{57,64,65}$ we also use the torus geometry later in Sec. IIIB for obtaining the ground state degeneracy at $5 / 2$ since the ground state degeneracy does not show 
up in the spherical geometry. The spherical geometry is defined by confining $N$ electrons to the surface of a sphere with a radial magnetic field produced by a magnetic monopole of strength $Q$ at the sphere center (the total flux through the sphere is $2 Q(h c / e)) . Q$ is an integer or half-integer due to Dirac's quantization condition and is related to the radius of the sphere through $R=\sqrt{Q}$. The filling factor in a particular LL with LL index $n$ is defined through its thermodynamic limit $\nu=\lim _{N \rightarrow \infty} N / g$ where $g=2(Q+n)+1$ is the total LL degeneracy. The total angular momentum $L$ is a good quantum number and a uniform state is the state with total angular momentum $L=0$ and is considered incompressible if it has a non-zero energy gap between the ground state and the low-lying excitation spectra.

We use the pseudopotentials calculated in the infinite planar geometry for carrying out our spherical system finite size diagonalization. The reasons for this are twofold: (1) it can be argued that the planar pseudopotentials, since they are the thermodynamic limit of the spherical pseudopotentials, better represent the real physical 2D system, and (2) it is much more convenient when considering finite-thickness modeling potentials to use planar pseudopotentials. At any rate, as discussed below, it makes very little difference whether we use planar or spherical pseudopotentials, and our conclusions (although perhaps not the precise values of the overlap in each case) are completely independent of this approximation. We believe that all qualitative conclusions in this paper are independent of our planar pseudopotential approximation.

TABLE I: Overlap integrals between the exact ground state wavefunction using spherical and planar pseudopotentials $\left(\left\langle\Psi_{\text {sphere }} \mid \Psi_{\text {plane }}\right\rangle\right)$, respectively. Also given are the overlap between the Laughlin or Pfaffian wavefunction and the exact ground state wavefunction using spherical and planar pseudopotentials $\left(\left\langle\Psi_{a} \mid \Psi_{\text {plane }}\right\rangle\right.$ and $\left\langle\Psi_{a} \mid \Psi_{\text {sphere }}\right\rangle$ where $a$ denotes either $L$ for Laughlin or Pf for Pfaffian as appropriate). This table quantifies the similarities and differences between states using planar or spherical pseudopotentials.

\begin{tabular}{|c|c|c|c|c|}
\hline$N$ & $\nu$ & $\left\langle\Psi_{\text {sphere }} \mid \Psi_{\text {plane }}\right\rangle$ & $\left\langle\Psi_{a} \mid \Psi_{\text {plane }}\right\rangle$ & $\left\langle\Psi_{a} \mid \Psi_{\text {sphere }}\right\rangle$ \\
\hline 6 & $1 / 3$ & 0.998840 & 0.992129 & 0.996446 \\
\hline & $7 / 3$ & 0.948005 & 0.736947 & 0.528481 \\
\cline { 2 - 5 } & $13 / 3$ & 0.917779 & 0.021261 & 0.013854 \\
\hline \multirow{2}{*}{5} & $1 / 5$ & 0.999966 & 0.996919 & 0.997427 \\
\hline & $11 / 5$ & 0.999974 & 0.997886 & 0.998198 \\
\cline { 2 - 5 } & $21 / 5$ & 0.988710 & 0.000012 & 0 \\
\hline \multirow{2}{*}{8} & $1 / 2$ & 0.997841 & 0.895311 & 0.921297 \\
\hline \multirow{6}{*}{} & $5 / 2$ & 0.968754 & 0.963623 & 0.867392 \\
\cline { 2 - 5 } & $9 / 2$ & 0.978191 & 0.030311 & 0.002384 \\
\hline \multirow{2}{*}{10} & $1 / 2$ & 0 & 0.889655 & 0 \\
\hline \multirow{2}{*}{} & $5 / 2$ & 0.972034 & 0.934183 & 0.837637 \\
\cline { 2 - 5 } & $9 / 2$ & 0.986467 & 0 & 0 \\
\hline
\end{tabular}

For this work we are interested in the FQHE at filling factors $1 / 3,1 / 5$, and $1 / 2$ in the LLL, SLL, and TLL. As described in Sec. IIA all the calculations are done in the LLL and all information about higher LLs is encapsulated by the pseudopotentials $V_{m}^{(n)}$. The relationship between the LL degeneracy $g=2 l+1$ ( $l$ is the single-particle angular momentum ${ }^{66}$ ) and the number of electrons $N$ for these states is as follows: $\nu=1 / 3$ has $2 l=3 N-3, \nu=1 / 5$ has $2 l=5 N-5$, and $\nu=1 / 2$ has $2 l=2 N-3$. In general, the relation between $l$ and $N$ for some LL filling factor $\nu$ is $2 l=\nu^{-1} N+\chi$ where $\chi$ is referred to as the "shift". The shift is non-zero in the spherical geometry and a consequence of the finite curvature of the spherical surface-this is discussed in more detail in Sec. IIIB. The $(N, l)$ relation for $\nu=1 / 2$ was chosen to be the same as it is for the Pfaffian wavefunc$\operatorname{tion}^{33}$ and the $(N, l)$ for $\nu=1 / 3$ and $1 / 5$ was chosen to be the same as for the Laughlin wavefunction. We further note that the FQH states considered in the work are not so-called "alias" states, that is, the $(N, l)$ relations used only correspond to fillings $1 / 3,1 / 5$, or $1 / 2$ (and $2 / 3$ and $4 / 5$ through particle-hole symmetry). This somewhat restricts our choice of particle numbers in our finite size diagonalization and will be mentioned again in Sec. $\mathrm{V}$ in relation to the calculation of excitation gaps. We believe that the price of restricting $N$ in avoiding any aliasing problem is well worth paying in our work because our wavefunction overlap calculations become necessarily unique since no two distinct FQH states compete at the same $(N, l)$ values.

Confusion about notation can sometimes arise when considering FQH states in higher LLs. Here we clarify our conventions. In the LLL the $1 / 3,1 / 5$, and $1 / 2$ states exist at an experimental filling factor of $\nu=1 / 3, \nu=1 / 5$, and $\nu=1 / 2$, respectively. In the SLL, the LLL (both spin up and down) are filled and inert yielding filling factors for $1 / 3,1 / 5$, and $1 / 2$ of $\nu=2+1 / 3=7 / 3, \nu=11 / 5$, and $\nu=5 / 2$, respectively. Finally, for the TLL, both the LLL and SLL (both spin up and down) are filled and inert yielding, for $1 / 3,1 / 5$, and $1 / 2, \nu=4+1 / 3=13 / 3$, $\nu=21 / 5$, and $9 / 2$. Hence, it should be clear that the FQH state at filling $1 / 5$ in the TLL corresponds to an experimental $\nu=21 / 5$, for example, and so on.

Since we are using planar, instead of spherical, pseudopotentials we quantify the difference between the two. Table \provides a number of overlaps for the FQH states considered here for the pure 2D $(d=0)$ Coulomb interaction. What is clear from these results is that the overlap between the exact ground states using either the planar $\left(\left|\Psi_{\text {planar }}\right\rangle\right)$ or spherical $\left(\left|\Psi_{\text {sphere }}\right\rangle\right)$ pseudopotentials (Table I third column) are generally high $\left(\left\langle\Psi_{\text {sphere }} \mid \Psi_{\text {plane }}\right\rangle>0.9\right)$. The major exception is the $N=10$ electron system. Here the symmetry of the ground state at filling factor $1 / 2$ in the LLL is different, on the sphere versus on the plane, yielding a vanishing overlap. It is for this reason 67 that we do not consider the $N=10$ electron system in this work. Furthermore, the overlap for either the Laughlin or the Pfaffian state 


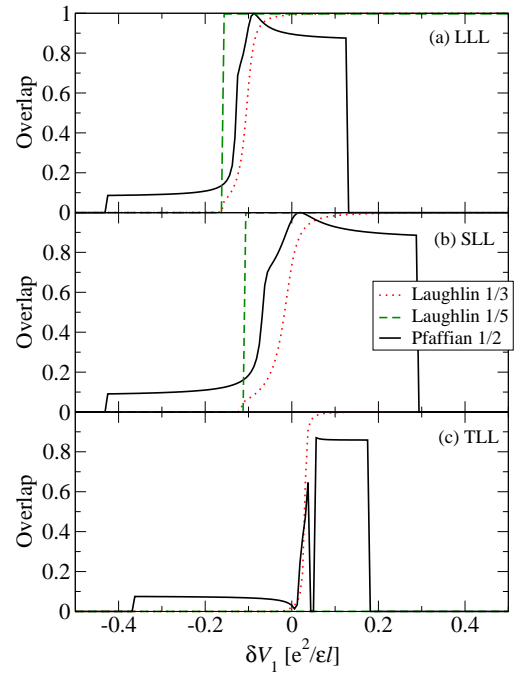

varying $V_{1}$

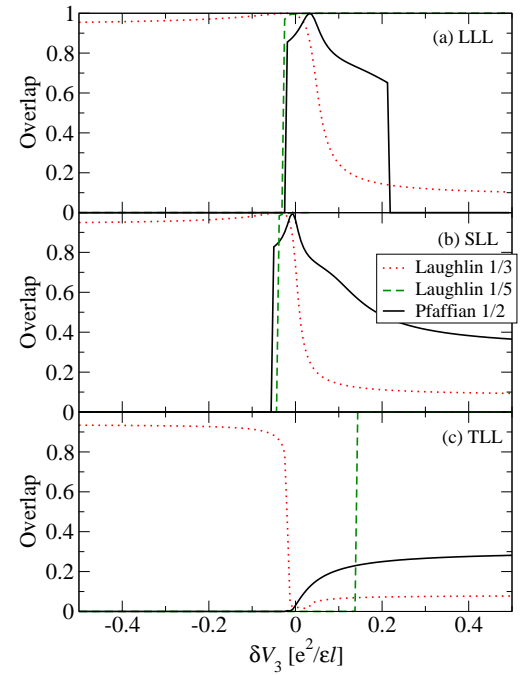

varying $V_{3}$

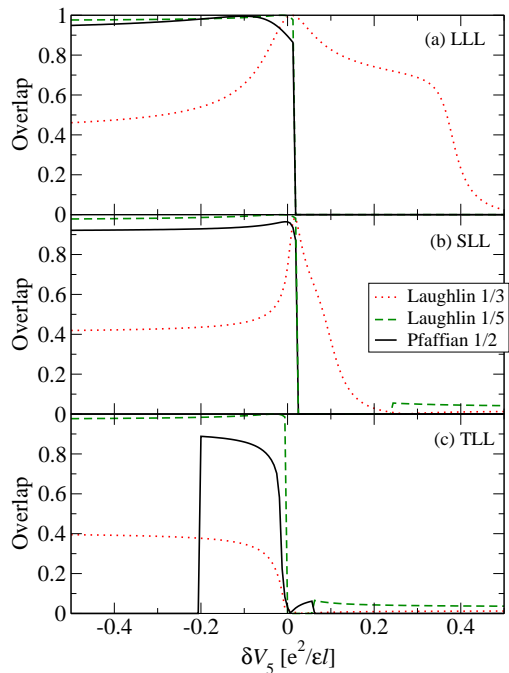

varying $V_{5}$

FIG. 6: (Color online) Overlaps of either the Laughlin or the Pfaffian wavefunction with the exact ground state wavefunction of the pure $(d=0)$ Coulomb Hamiltonian where $V_{1}$ (left), $V_{3}$ (middle), and $V_{5}$ (right) are varied by $\delta V_{1}, \delta V_{3}$, and $\delta V_{5}$, respectively. Of course, $\delta V_{i}=0$ corresponds to the "Coulomb point" where the interaction is a pure 2D Coulomb interaction. In the above figures, the situations considered are $\nu=1 / 3$ for $(N, l)=(6,7.5)$ (dotted line), $\nu=1 / 5$ for $(N, l)=(5,10)($ dashed line), and $\nu=1 / 2$ for $(N, l)=(8,6.5)$ (solid line).

between the exact planar and spherical states is qualitatively similar (Table \columns four and five). One point of note is $N=5$ at $\nu=21 / 5(1 / 5$ in the TLL); here the overlap with the Laughlin state and the exact planar state is nearly zero (0.000012) but retains the same symmetry. However, the overlap with the Laughlin state and the exact spherical state is exactly zero due to a different symmetry. None of these minor technical issues has any bearing on our main goal in this work, which is to study the comparative qualitative trends of the quasi-2D finite layer thickness effect on the stability of the Laughlin or the Pfaffian FQH state at $\nu=1 / 5,1 / 3$, and $1 / 2$ in the lowest three orbital Landau levels. In particular, we want to study how the finite thickness of the quasi-2D experimental systems affects the comparative stability of the Laughlin (for $\nu=1 / 3$ and $1 / 5$ ) and the Pfaffian $(\nu=1 / 2)$ state in different $(n=0,1,2)$ orbital Landau levels.

We now return to the calculation of overlaps. In the fourth column of Table \ the calculated overlap between the exact state (using planar pseudopotentials) at filling factor $1 / 3,1 / 5$, and $1 / 2$ and the Laughlin $(1 / 3$ and $1 / 5)$ and the Pfaffian $(1 / 2)$ states, respectively, for the pure $2 \mathrm{D}(d=0)$ system in the LLL, the SLL, and the TLL is shown for $N=5,6,8$, and 10 . In the LLL the overlap at $1 / 3$ and $1 / 5$ is very high $(\sim 0.99)$. For $1 / 2$, however, the overlap is not nearly as high $(\sim 0.9)$. An overlap of 0.9 or less is not considered particularly compelling in the FQHE and is indicative of, perhaps, different physics. In fact, experimentally there is no FQHE observed in the LLL at $1 / 2$ to date. In the SLL the story changes.
For $1 / 3$ the overlap is significantly decreased $(\sim 0.74)$ while for $1 / 5$ the overlap stays as high as it is in the LLL $(\sim 0.99)$. The overlap at $1 / 2$ increases in the SLL compared to the LLL to a respectable value $(\sim 0.96)$. In the TLL the overlap at $1 / 3,1 / 5$, and $1 / 2$ is essentially zero although the symmetry between the two remains the same. The most straightforward conclusion following from the $d=0$ results shown in Table $\square$ is that the Laughlin state is the stable FQH state at $\nu=1 / 3$ and $1 / 5$ in the LLL even in the strict $2 \mathrm{D}$ limit, but the other FQH states may not exist in the ideal 2D limit except for $\nu=1 / 5$ in the SLL.

A theoretical strategy often used in studying the FQHE is to vary the first few pseudopotentials away from the ideal 2D Coulomb values, in an ad hoc way, to investigate whether the overlap between the resulting exact states and the Laughlin or Pfaffian states gets better or worse. In Fig. 6 we consider a Hamiltonian where we have varied $V_{1}, V_{3}$, and $V_{5}$ independently symmetrically around the Coulomb point in the LLL (left panel), SLL (middle panel), and TLL (right panel) (the results at the Coulomb point are, of course, given in Table 【).

Clearly, changes in $V_{1}, V_{3}, V_{5}$ that bring the effective $\hat{H}$ closer to $\hat{H}_{L}^{(3)}\left(\hat{H}_{L}^{(5)}\right)$ increase the overlap between the Laughlin wavefunction at $1 / 3(1 / 5)$ and the exact wavefunction, while changes opposite to this decrease the overlap. These conditions are obtained, for example, when $V_{1}$ is increased producing a high overlap for $1 / 3$ and $1 / 5$ in the LLL and SLL (Fig. 6 left panel (a) and (b)). (In the TLL, however, $1 / 5$ has a zero overlap due to a symmetry change.) Increasing $V_{3}$ takes $\hat{H}$ into 
$\hat{H}_{L}^{(5)}$ which is evident by the very high overlap between the $1 / 5$ Laughlin state and the exact state in LLL, SLL, and TLL ((a)-(c) in the middle panel of Fig. 6). Nothing particularly non-trivial is happening here because we know how $\hat{H}$ is connected to $\hat{H}_{L}^{(q)}$, i.e., any changes in $\hat{H}$ towards (away from) $\hat{H}_{L}^{(q)}$ makes the Laughlin state a better (worse) description for the 1/q FQHE state.

The overlap variation with changing $V_{1}, V_{3}, V_{5}$ for the Pfaffian state $(\nu=1 / 2)$ apparent in Fig. 6] cannot, however, be explained easily since there is no existing twobody Hamiltonian, e.g., $\hat{H}_{L}^{q}$ for the Laughlin state, for which the Pfaffian is an exact eigenstate. It is clear (and has been shown previously ${ }^{35,39}$ ) that in the LLL and SLL changing $V_{1}$ and $V_{3}$ in particular ways can produce a state with an overlap close to unity for the Pfaffian, i.e., Fig. 6 (a) and (b) in the left and middle panel. It is also interesting to note that in the SLL the value of $\delta V_{1}$ and $\delta V_{3}$ that gives this high overlap between the Pfaffian and the exact state at $\nu=1 / 2$ is very near the Coulomb point. Changing $V_{5}$ (right panel) can also produce a high overlap for the Pfaffian. Qualitatively it behaves similarly to the $1 / 5$ Laughlin state as $V_{5}$ is decreased in the LLL and SLL. The Pfaffian never achieves a particularly high overlap in the TLL for any values of $V_{1}, V_{3}, V_{5}$ we looked at.

However, the technique of changing $V_{m}$ 's is arbitrarily artificial and ad hoc, without shedding much light on how real experimental quasi-2D systems, where $V_{m}$ 's are determined by the layer thickness $d$, will behave. (In fact, all the $V_{m}$ 's change when considering finite thickness, a fact that is further investigated in Sec. IV this means that tuning just one specific $V_{m}$, e.g., $V_{1}$ or $V_{3}$ or $V_{5}$, as done in Fig. 6 is purely a theoretical construct which is impossible to achieve in real $2 \mathrm{D}$ systems.) To understand the variation in the states as a function of $d$ and their incompressible or compressible nature we carefully define the overlap calculated previously in Ref. 19 which is $\left\langle\Psi_{L(P f)} \mid \Psi_{\nu}(d)\right\rangle$. This overlap quantifies exactly how similar $\left|\Psi_{\nu}(d)\right\rangle$ is to the Laughlin wavefunction $\left|\Psi_{L}\right\rangle$ $(\nu=1 / 3$ or $1 / 5)$ or the Pfaffian wavefunction $\left|\Psi_{P f}\right\rangle$ $(\nu=1 / 2)$ as a function of the finite layer thickness $d$. We have some intuition (and previous results ${ }^{19}$ ) about how the overlap will behave with increasing $d$ after studying the $f$ - and $g$-functions (cf. Sec. IIC). In Figures 7 , 8 , and 9 we report overlaps in the LLL (left panel), SLL (middle panel), and the TLL (right panel) of the Laughlin or Pfaffian wavefunction and the exact wavefunction for the finite-thickness models of ZDS, SQ, and FH for fillings $1 / 3,1 / 5$, and $1 / 2$, respectively. We emphasize that the variation in $d$ cannot be described in terms of a variation in the value of one (or a few) pseudopotentials $\left(V_{m}\right)$.

In Figs. 7, 8, 9, 10, and 14 we normalize $d$ defining a new width parameter $d$ ' such that all the "widths" for each potential are defined equivalently. For the SQ and FH confinement (as well as the PQW confinement) one can calculate $w=\sqrt{\left\langle z^{2}\right\rangle-\langle z\rangle^{2}}$ which is the variance of the wavefunction (i.e., the root-mean-square fluctuation in the electron position). Using the values of $w$ for each potential, $w_{S Q}$ for the SQ and $w_{F H}$ for FH, we normalize the FH potential to the SQ potential. That is, we rescale $d$ in the $\mathrm{FH}$ potential to $d^{\prime}=\left(w_{F H} / w_{S Q}\right) d=$ $(0.57735 / 0.180756) d$, while $d^{\prime}$ for the SQ confinement is just the original $d$ in that model (since $w_{S Q} / w_{S Q}=1$ ). To rescale the ZDS potential we use a more ad hoc but well defined method. Using $\nu=1 / 2$ in the SLL we scale the maximum in the overlap between the exact wavefunction at $d$ and the Pfaffian wavefunction for the ZDS potential to be equal to the maximum using the SQ potential, i.e., $d^{\prime}=(4.6 / 1.4) d$. In this way the behavior of the overlap as a function of $d^{\prime}$ for each finite-thickness potential is quantitatively similar. Further, in Figs. 7, 8, 9, 10, and 14 we also give the overlaps as functions of $w$ in units of magnetic length. With this parameterization one is able to distinguish between two regimes of layer thickness, i.e., $w / l<1$ and $w / l>1$. Note that our rescaling of the width parameter from $d$ to $d^{\prime}(\propto w)$ is a purely nonessential book-keeping procedure which makes it explicit that, when the quasi-2D width parameter is properly defined (i.e., $d^{\prime}$ ), then the different quasi-2D models show similar quantitative trends in the calculated overlaps as a function of layer width. Theoretical descriptions in terms of $d$ or $d^{\prime}$ are completely equivalent-the only advantage of using the normalized thickness parameter $d^{\prime}$ is that the calculated overlap is now quantitatively similar in all the quasi-2D models we consider.

For the sake of completeness we provide below the formulae for $w=\sqrt{\left\langle z^{2}\right\rangle-\langle z\rangle^{2}}$ and $d^{\prime}$ for the four models (SQ, FH, ZDS, and PQW) respectively in terms of their wavefunction parameter, i.e., $d$, as given in Eqs. 7. 9] (and Eq. 18)

$$
\begin{aligned}
w_{S Q} & =0.180756 d \\
w_{F H} & =0.57735 d \\
w_{P Q W} & =0.5 d \\
d_{S Q}^{\prime} & \equiv \frac{w_{S Q}}{w_{S Q}} d=d \\
d_{F H}^{\prime} & \equiv \frac{w_{F H}}{w_{S Q}} d=3.194085 d \\
d_{S Q}^{\prime} & \equiv \frac{w_{P Q W}}{w_{S Q}} d=2.76616 d \\
d_{Z D S}^{\prime} & \equiv \frac{4.6}{1.4} d=3.285714 d
\end{aligned}
$$

Note that for the ZDS model there is no single particle wavefunction in the $z$-direction, $\eta(z)$, that produces the effective potential of the form of the ZDS model, hence, we cannot define $w_{Z D S}$, and $d^{\prime}$ is calculated as described above.

\section{Filling factor $1 / 3$ (Laughlin wavefunction)}

We first concentrate on filling factor $1 / 3$ (Fig. 17). In the LLL (left panel) the overlap between the Laughlin 


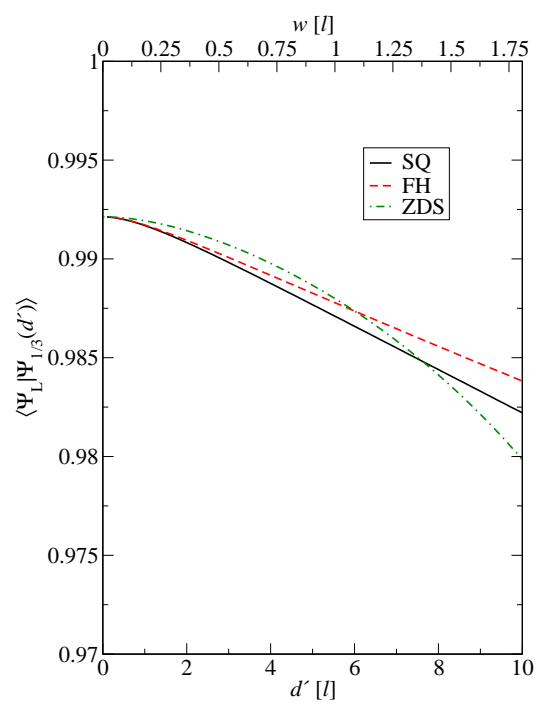

(a) LLL

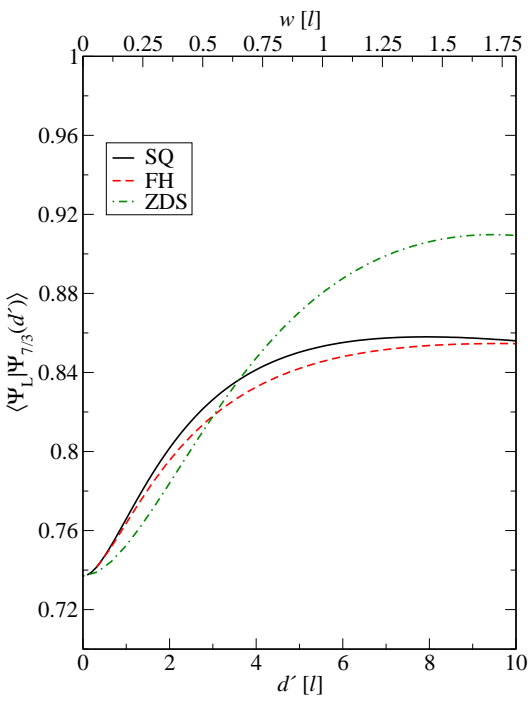

(b) SLL

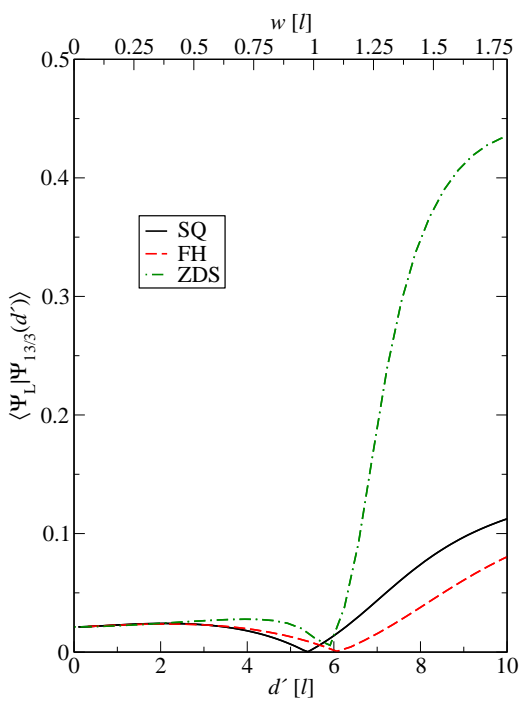

(c) TLL

FIG. 7: (Color online) Overlap $\left(\left\langle\Psi_{L} \mid \Psi_{\nu}(d)\right\rangle\right)$, as a function of thickness $d^{\prime}$, between the Laughlin and the exact wavefunctions at the fractional filling $1 / 3$ in the $n$-th LL, i.e., the LLL $(\nu=1 / 3)($ left $)$, the SLL $(\nu=7 / 3)($ middle), and the TLL $(\nu=13 / 3)$ (right). The overlap as a function of $w$ is also given on the horizontal axis at the top of each plot. The particular system has $N=6$ electrons at $l=7.5$. The different curves represent the different finite-thickness modeling potentials: SQ (solid line), FH (dashed line), and ZDS (dashed-dotted line). Note the different scale in each plot of the vertical axis.

state and the exact state decreases as $d^{\prime}$ increases. In fact, this was previously shown for the ZDS potential in Ref. 19. The SQ and FH potentials also show a decrease in overlap for increasing $d^{\prime}$ but the change is mild compared to the ZDS potential in that the overlap drops from near unity only to $\sim 0.975-0.96 \underline{68}$. Qualitatively, the finite-thickness could eventually destroy the FQHE at $1 / 3$ in the LLL, again, as first reported in Ref. 19. But our results tend to support a scenario where such a destruction is continuous as a function of $d^{\prime}$, not abrupt at any particular value of $d^{\prime}$. More specifically, our finite system study, as shown in Figs. 7.9 does not indicate the occurrence of a thickness-driven sharp transition from an incompressible FQH state to a compressible one-any such transition seems continuous, although in practice the excitation gap may become very small for large thicknesses.

In the SLL (middle panel of Fig. (7) the overlap starts at a modest value $(\sim 0.74)$ (cf. Table I) for $d^{\prime}=0$ and an increase in overlap is seen for increasing $d^{\prime}$ until a maximum is obtained for large $d^{\prime}$ (increasing $d^{\prime}$ to unrealistic values produces an overlap that approaches zero). We note that the value of $d^{\prime}$ where the highest overlap is obtained corresponds to $w / l \approx 1$ which, perhaps, more clearly shows the effect of the layer thickness, i.e., the overlap is seen to decrease as $w / l$ moves away (negatively or positively) from unity-this qualitative feature is again seen when filling factor $1 / 2$ in the SLL is investigated, cf. Fig. 9] This result could be anticipated from the observation that the $f$-functions for the SLL have a local minimum for non-zero $d$ (or scaled $d^{\prime}$ ). However, it should be noted that the overlap, while increasing for non-zero $d^{\prime}$, still only reaches a modest value of approximately 0.84-0.92 (depending on the quasi-2D model).

In the TLL (right panel of Fig. 7) the overlap never reaches a value greater than 0.5 (for any model) indicating that the Laughlin state is not a good description of the exact state in the TLL. Again, this result is evident in the Laughlin-unfriendly nature of the $f$-functions in the TLL.

\section{Filling factor 1/5 (Laughlin wavefunction)}

Next we consider filling factor $1 / 5$ in the LLL (left panel), SLL (middle panel), and TLL (right panel) shown in Fig. 8. From an investigation of the $g$-functions we would expect the $1 / 5$ Laughlin state to have a high overlap with the exact state for a large range of $d^{\prime}$ in the LLL and SLL and that is, in fact, what can been seen in Fig. 8. In the LLL and SLL the overlap is greater than 0.995 for all models up to large $d^{\prime}$ (the overlap in the LLL and SLL appear nearly identical in their qualitative and quantitative behavior). This result indicates that the $1 / 5 \mathrm{FQH}$ state is as strong in the SLL as it is in the LLL, which is consistent with earlier results $47,48,49,69$. Note that nothing interesting happens to the overlap in any of the first three LLs at $w / l \approx 1$.

The TLL provides a strange scenario for $1 / 5$. Here the exact ground state has a different symmetry from the Laughlin state at $d^{\prime}=0$ yielding a vanishing overlap. However, as $d^{\prime}>0$ the overlap very abruptly becomes non-zero and large $(\sim 0.95)$. Again, large $d^{\prime}$ reduces the 


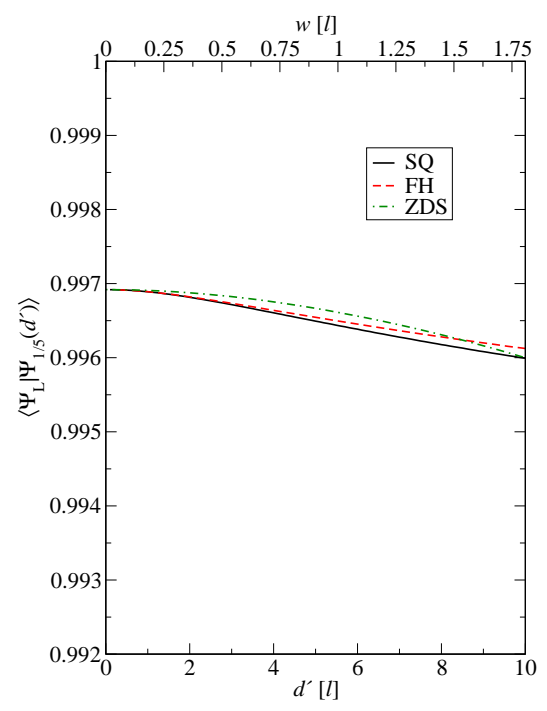

(a) LLL

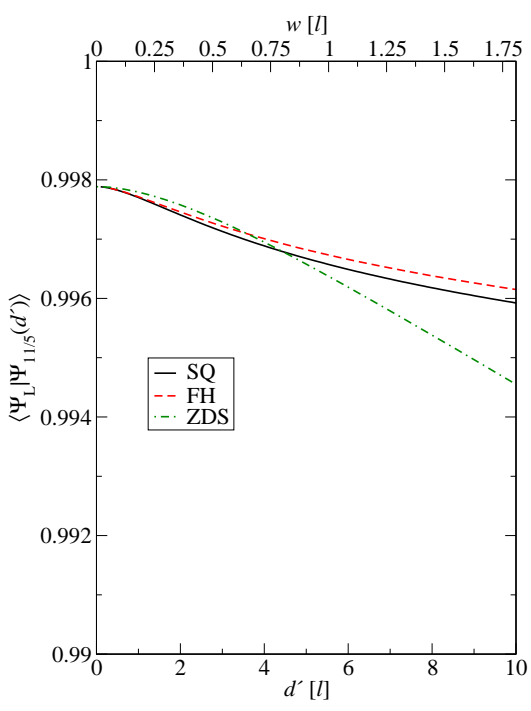

(b) SLL

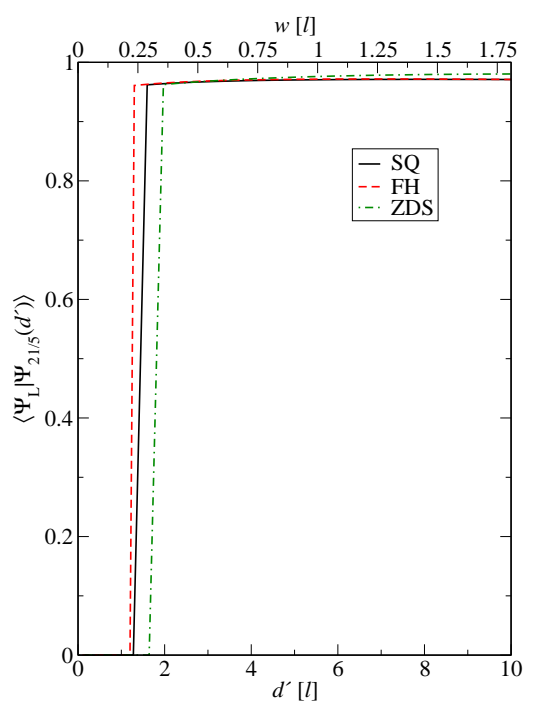

(c) TLL

FIG. 8: (Color online) Same as Fig. 7 except for fractional filling 1/5. The particular system has $N=5$ electrons at $l=10$.

overlap severely for the ZDS potential (not shown) while only moderately for the SQ and FH potentials (although in the reported range of $d^{\prime}$, on this scale, the overlap appears essentially constant).

Note that the qualitative behavior of our calculated overlap for filling factor $1 / 3$ and $1 / 5$ in the SLL is different in that for $1 / 3$ increasing $d^{\prime}$ improves the overlap while for $1 / 5$ increasing $d^{\prime}$ worsens the overlap, as it does in the LLL. Based on our overlap calculation we would therefore predict a more (less) stable $7 / 3(11 / 5)$ FQHE with increasing quasi-2D layer thickness.

\section{Filling factor 1/2 (Pfaffian wavefunction)}

Lastly, and most importantly, we turn to filling $1 / 2$ in the LLL (left panel), SLL (middle panel), and TLL (right panel) in Fig. 9, considering now the overlap between the Moore-Read Pfaffian wavefunction and the exact numerical wavefunction at $\nu=1 / 2$. In the LLL, the overlap starts at approximately 0.9 for $d^{\prime}=0$. However, unlike the LLL behavior for $1 / 3$ and $1 / 5$ where the overlap monotonically decreases as $d^{\prime}$ increases, the overlap between the Pfaffian and the exact wavefunction here increases to a weak maximum for some finite $d^{\prime}$. The maximum overlap for the ZDS, SQ, and FH potentials is moderate and barely above the $d^{\prime}=0$ value of approximately 0.9 , however. Nevertheless, there is a distinct quasi-2D width induced enhancement of the Pfaffian overlap here at $\nu=1 / 2$, not apparent in the corresponding $\nu=1 / 3$, and $1 / 5$ Laughlin states in the LLL where the overlap decreases monotonically with increasing layer thickness.

In the SLL the overlap of the Pfaffian wavefunction with the exact wavefunction increases from 0.96 for $d^{\prime}=0$ to essentially unity for finite $d^{\prime} / l \sim 5$. This result sug- gests that the finite layer thickness of the quasi-2D experimental system actually leads to an exact wavefunction that is more like the Pfaffian at $\nu=5 / 2$ for finite $d^{\prime}$ than for $d^{\prime}=0$. Continually increasing $d^{\prime}$ beyond this optimal value produces wavefunctions with decreasing overlaps (similar to $1 / 3$ and $1 / 5$ ). This finite width induced stabilization of the Pfaffian state at $\nu=5 / 2$ perhaps explains the fragility of the observed $5 / 2$ FQHE in experiments.

In both the LLL and SLL we see that the highest $\nu=$ $1 / 2$ Pfaffian overlap occurs for $w / l \approx 1$. This is similar to what was observed for filling factor $1 / 3$ in the SLL (Fig. 7) but the effect is more pronounced at $1 / 2$.

Filling factor $1 / 2$ in the TLL behaves much the same as for $1 / 3$. The overlap starts below 0.05 for $d^{\prime}=0$ and achieves a value which is model dependent, between 0.125-0.25 for some finite $d^{\prime}$ (for very large $d^{\prime}$ all overlaps eventually approach zero). From this calculation one would not expect to see the FQHE at $\nu=1 / 2$ in the TLL.

The preceding calculations establish that including finite layer thickness of the realistic quasi-2D system produces non-trivial behavior of the overlap between either the Laughlin or Pfaffian wavefunction with the exact wavefunction. The physical expectation based on our overlap calculation would be that the FQHE in the SLL is stronger for the $1 / 5$ and $1 / 2$ state (provided there is some finite $d^{\prime}$ ) than it is for the $1 / 3$ state. Experimentally $1 / 3,1 / 5$, and $1 / 2$ (and their particle-hole conjugates) are all observed in the SLL 15,16 .

To make a connection between experiment and theory we consider the recent experimental observations of $\nu=5 / 2$ reported by Choi, et al. $\frac{15}{\sqrt{15}}$ and Pan, et al $\stackrel{16}{ }^{16}$ which were obtained in quantum well structures of width $30 \mathrm{nms}$. In Ref. 15 and $16 \nu=5 / 2$ was observed at a $B$ field of approximately $B \approx 4.6 \mathrm{~T}$ and $5.2 \mathrm{~T}$, respec- 


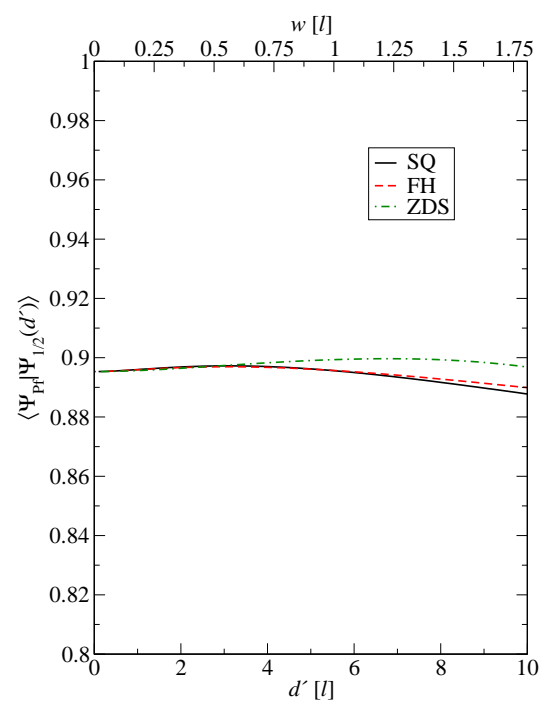

(a) LLL

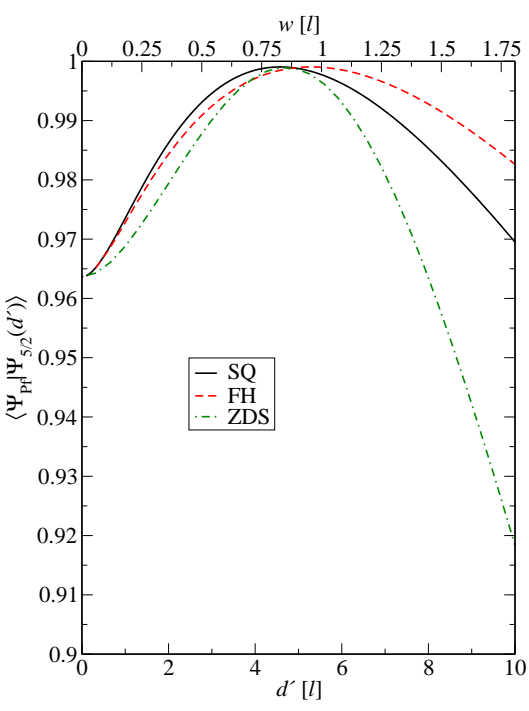

(b) SLL

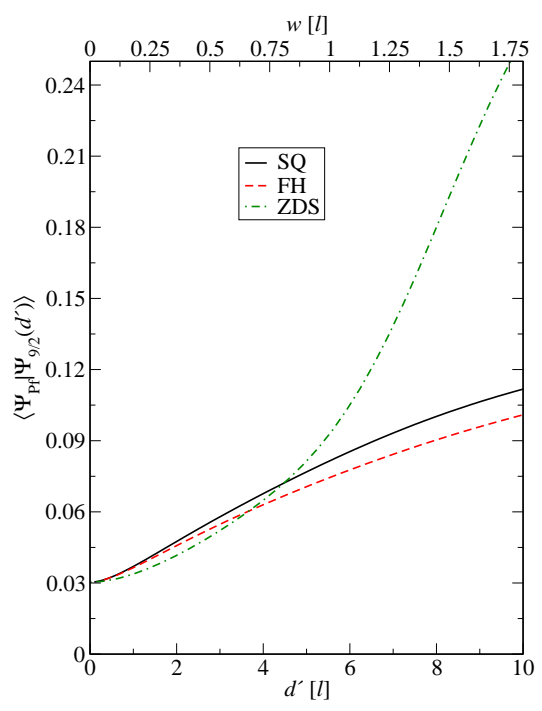

(c) TLL

FIG. 9: (Color online) Same as Figs. 7 and 8 except for fractional filling 1/2. Hence, the relevant overlaps are between the exact ground state wavefunction at some thickness $d^{\prime}\left(\left|\Psi_{\nu}(d)\right\rangle\right)$ and the Pfaffian wavefunction $\left(\left|\Psi_{P f}\right\rangle\right)$. The particular system has $N=8$ electrons at $l=6.5$.

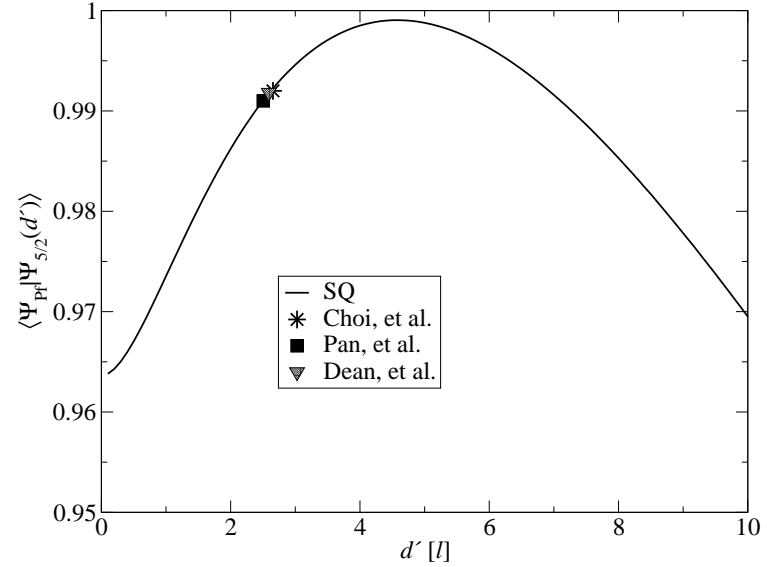

FIG. 10: Same as Fig. 9 (middle panel, SLL) except only the SQ potential is shown. The experimental $d^{\prime}$ of Refs. 15 and 16 are shown as a asterisk and solid square, respectively. The particular system has $N=8$ electrons at $l=6.5$. Also show is the result for a very recent experiment (Ref. 85) by Dean, et al.

tively. Using the standard formula for magnetic length in GaAs/AlGaAs quantum wells $(l \approx 25 \mathrm{~nm} / \sqrt{B[\mathrm{~T}]})$ we plot in Fig. 10 the value of $d^{\prime}$ these particular experiments correspond to on our overlap plot at $1 / 2$ in the SLL $(\nu=5 / 2)$ for the SQ potential. From this comparison we see that the experimental systems are not optimized to observe the strongest possible Pfaffian state at $\nu=5 / 2$. Somewhat increasing the value of the quantum well width so that $d^{\prime} / l$ (or equivalently $d / l$ ) is in- creased to the optimal value should give more a stable $5 / 2$ FQHE! This is further elaborated (and reinforced) by studying the ground state topological degeneracy on a torus, a defining hallmark of non-Abelian states in the next section.

\section{B. Threefold topological degeneracy signature of the Pfaffian state on the torus}

Recall (cf. Sec. IIA that in the spherical geometry, the signature of an incompressible FQH state is the existence of a rotationally symmetric uniform state with total angular momentum $L=0$ with a finite excitation gap to higher energy states. A given FQH state generally has a "shift" in the equation relating the number of electrons $N$ to the total flux $2 Q$ through the finite sphere. The MR Pfaffian wavefunction is written on the sphere as

$$
\Psi_{P f}=\operatorname{Pf}\left(\frac{1}{u_{i} v_{j}-u_{j} v_{i}}\right) \prod_{i<j}\left(u_{i} v_{j}-u_{j} v_{i}\right)^{2},
$$

where the spinor coordinates are $u_{j}=\cos \left(\theta_{j} / 2\right) \mathrm{e}^{-i \phi_{j} / 2}$ and $v_{j}=\sin \left(\theta_{j} / 2\right) \mathrm{e}^{i \phi_{j} / 2}$ with $(\theta, \phi)$ being the coordinates on the surface of a sphere. The Pfaffian symbol above corresponds to

$$
\operatorname{Pf}\left(A_{i j}\right)=\sum_{\sigma} \epsilon_{\sigma} A_{\sigma(1) \sigma(2)} \ldots A_{\sigma(N-1) \sigma(N)},
$$

where $\sigma$ are permutations of the $\mathrm{N}$ particle indices. It is found that the wavefunction in Eq. 12 requires a flux $2 Q=2 N-3$. While this corresponds to filling factor 
$1 / 2$ in the thermodynamic limit $(N \rightarrow \infty)$ the nontrivial constant shift of -3 in this relation is characteristic of the Pfaffian state and arises from the curvature of the spherical surface. For example, a possible competing state at $\nu=1 / 2$ is the composite fermion Fermi sea ${ }^{70}$ with $2 Q=2 N-2$. While the ability to discern between competing states via the "shift" may be considered an advantage of the spherical geometry, it also has the drawback that one cannot directly address the competition between different phases without moving to a different Hilbert space with a different flux. (In addition, complications may also arise from distinct thermodynamic FQH states occurring at the same shift for given $(Q, N)$ values-the so-called 'aliasing' problem.)

This problem is resolved using the torus geometry where the shift is zero and all states are uniquely defined by the filling factor alone. Thus, exact diagonalization on the torus provides additional information on the nature of the ground state. We have performed calculations in periodic rectangular domains with unequal sides $a$ and $b$. In the presence of a magnetic field, the standard translation operators no longer commute but they do satisfy the so-called magnetic translation algebra. This noncommutation of the standard translation operators prevents a simple straightforward construction of conserved quantum numbers. Haldane ${ }^{71}$ has, however, shown how to construct many-body states that have conserved pseudomomenta corresponding to the magnetic translations along the two periodicity directions. These pseudomomenta are bi-dimensional $\left(K_{x}, K_{y}\right)$ and they reside in a two-dimensional Brillouin zone containing exactly $N_{0}^{2}$ points where $N_{0}$ is the greatest common divisor of $N$ and $N_{\phi}$, where $N_{\phi}$ is the number of flux quanta through the system (here we denote the total flux as $N_{\phi}$ compared to $2 Q$, as in the spherical geometry, to distinguish the geometries more readily and because $N_{\phi}$ is more commonly used in the torus geometry literature). The pseudomomenta are of the form $K_{x}=2 \pi \hbar s / a$ and $K_{y}=2 \pi \hbar t / b$ with $s, t=0, \ldots, N_{0}$.

On the torus, there is a degeneracy due to the center of mass motion given by $q$ at a filling factor $p / q$. In the construction of Haldane, the Hamiltonian is block-diagonal with exactly $q$ identical blocks-this holds independently of the Hamiltonian and, hence, it has nothing to do with the physics of the system. We have systematically discarded this trivial degeneracy in all that follows. The construction of conserved quantities has the practical advantage that it reduces the size of the Hilbert space in which we search-through exact diagonalization-for the few low-lying eigenstates.

At least some of the candidate states for a half filled LL have characteristic signatures in the quantum numbers of these low-lying eigenstates. In the case of the composite fermion Fermi sea, the effective theory is that of quasi-free fermions with an interaction induced mass. In a finite system, with discrete energy levels, one expects to find closed shell effects which, in addition, should be quite sensitive to the aspect ratio of the unit cell. This is what is observed 35 in the LLL at $\nu=1 / 2$. There are also other competing (compressible) phases with broken translational symmetry $72,73,74$ which are close in energythe so-called stripe and bubble phases. The stripe phases in the LLL are equivalent to charge-density waves with unidimensional electronic density modulation. The spectral signature of such a phase is a set of low-lying states with pseudomomenta all related by a single wavevector which has the periodicity of the stripe. In the SLL, Rezayi and Haldane $\mathrm{S}^{35}$ have numerically shown that such a stripe phase is the ground state for electrons interacting with the pure Coulomb potential with zero width.

On the other hand, the Pfaffian state has a very different spectral signature which can be obtained by translating the wavefunction (Eq. 12) in the torus geometry. One of the key ingredients of the Pfaffian is the LaughlinJastrow correlation factor $\prod_{i<j}\left(u_{i} v_{j}-u_{j} v_{i}\right)$ which, if the coordinates on the torus are $z=x+i y$, can be written as $\prod_{i<j}\left(z_{i}-z_{j}\right)$. The correlation factor with the proper periodicity is given by $\theta_{1}\left(z_{i}-z_{j} \mid \tau\right)$ with $\tau=i b / a(b / a$ is the aspect ratio of the rectangle) and $\theta_{1}$ the first Jacobi theta function. This allows a construction ${ }^{75}$ of the standard Laughlin state for filling factor $1 / m$ (with an additional factor to treat the center of mass motion).

This same construction does not work for the Pfaffian state. Indeed, the presence of a denominator in the formula (Eq.12) invalidates the periodicity properties if one simply introduces $\theta_{1}\left(z_{i}-z_{j} \mid \tau\right)$ factors. The correct procedure ${ }^{76.77}$ involves the four Jacobi theta functions $\theta_{1}$, $\theta_{2}, \theta_{3}$, and $\theta_{4}$ through the following substitution,

$$
\frac{1}{\left(z_{i}-z_{j}\right)} \rightarrow \frac{\theta_{a}\left(z_{i}-z_{j} \mid \tau\right)}{\theta_{1}\left(z_{i}-z_{j} \mid \tau\right)}, \quad \mathrm{a}=2,3,4 .
$$

This construction gives three ground states instead of only one in the spherical geometry. This remarkable degeneracy is of topological origin.

If one considers a physical Hamiltonian whose ground state is adiabatically connected to the Pfaffian state one expects to find the threefold degenerate multiplet whose quantum numbers on the torus may be deduced from Eq. 14. In a finite system, there will be a splitting of the multiplet of states, and it is only in the thermodynamic limit that these states will become truly degenerate. For electrons at filling $1 / 2$ the three Pfaffian ground states have pseudomomenta $\mathbf{K}=$ $\left(0, N_{0} / 2\right),\left(N_{0} / 2,0\right),\left(N_{0} / 2, N_{0} / 2\right)$ (in units of $2 \pi \hbar / a$ and $2 \pi \hbar / b)$ and this set of quantum numbers clearly differentiates the Pfaffian from the other ground states mentioned above. Hence, to probe for this quasi-degeneracy one has to use a rectangular unit cell since, in that case only, the degeneracy is non-trivial-square or hexagonal cells have additional discrete (geometric) symmetries leading to the equivalence of some or all Brillouin zone points potentially masking the non-Abelian topological degeneracy.

To fully understand the properties of the Pfaffian state, one further has to take into account the fact that it breaks particle-hole $(\mathrm{p}-\mathrm{h})$ symmetry $\stackrel{78,79}{ }$, either due to 

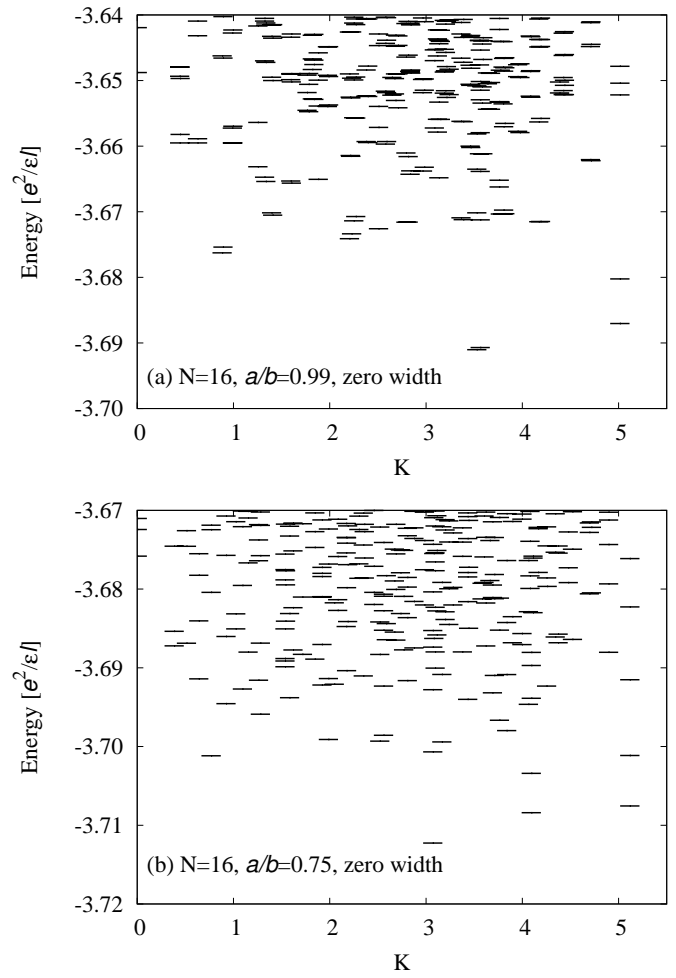

FIG. 11: Exact energy in units of $e^{2} / \epsilon l$ (only the low energy sector is being shown) versus pseudomomentum $\mathrm{K}=$ $\sqrt{(a / b) K_{x}^{2}+(b / a) K_{y}^{2}}$ for $N=16$ electrons, using the torus geometry, interacting via the SLL Coulomb Hamiltonian (zero width). The pseudomomenta $K_{x}$ and $K_{y}$ are given in units of $2 \pi \hbar / a$ and $2 \pi \hbar / b$, respectively. Panel (a) corresponds to an aspect ratio of the rectangular unit cell equal to 0.99 while (b) has an aspect ratio of 0.75 .

explicit p-h symmetry breaking terms present in real systems (e.g., Landau level mixing or coupling) or due to a spontaneous $\mathrm{p}$-h symmetry breaking in theories neglecting LL mixing ${ }^{80}$. If we consider the wavefunction written on the sphere, its flux $2 Q=2 N-3$ leads automatically to the $\mathrm{p}$-h conjugate state (the so-called anti-Pfaffian) at $2 Q=2 N+1$, which, since it exists at a different flux for the same $N$, precludes any mixing between the states. On the torus these two states will mix, for finite size systems, which should lead to a lower energy symmetric Pfaffian-Anti-Pfaffian combination and a higher energy antisymmetric combination. This reasoning applies to each of the three states with differing K's expected on the torus, so we expect to find three doublets if we are, in fact, dealing with a system well described by the Pfaffian state, corresponding to a non-trivial topological degeneracy of 6 in the thermodynamic limit.

To investigate the spectral signature of the Pfaffian we have performed exact diagonalizations on the torus from $N=10$ to 16 electrons using the SLL Coulomb interaction with finite thickness modeled by the three quasi-2D confinement models used before-FH, SQ and
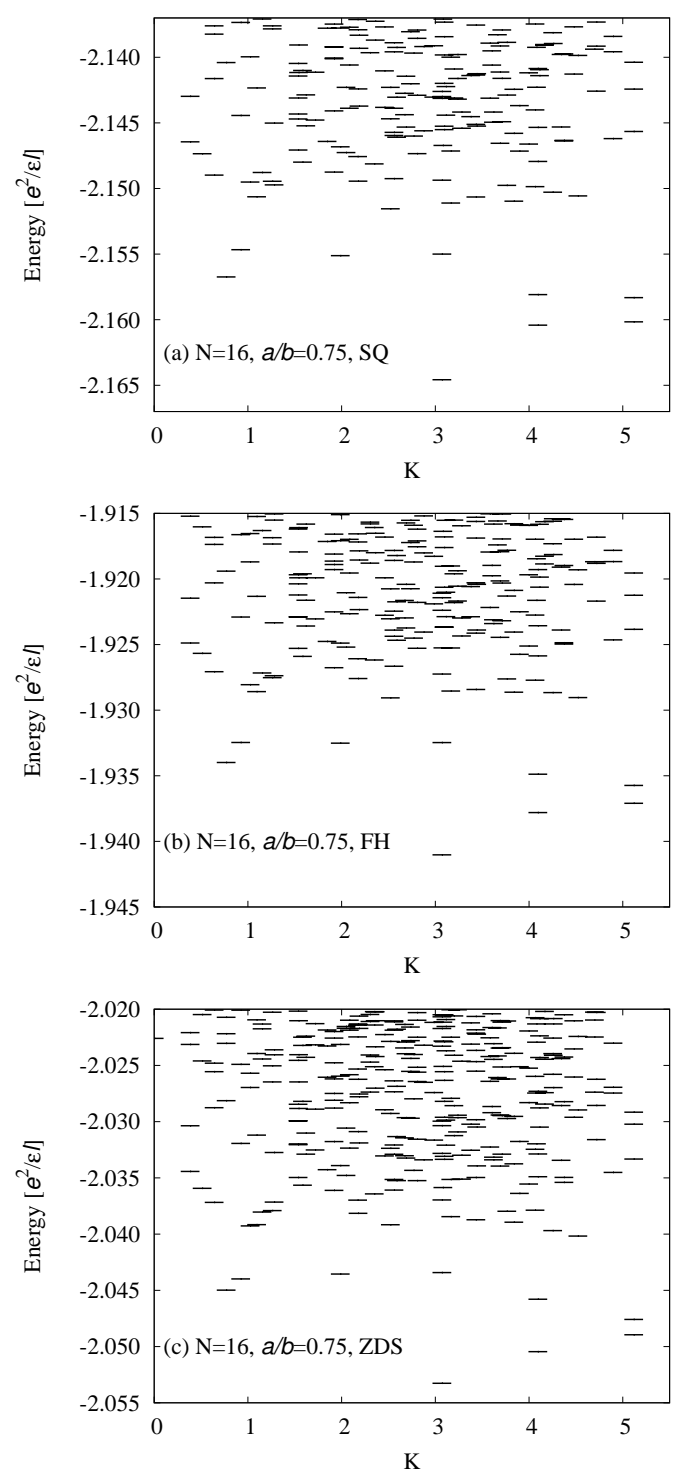

FIG. 12: Same as Fig. 11 except here we have included finite thickness effects using the SQ (a), FH (b), and ZDS (c) potentials, respectively. The aspect ratio is 0.75 .

\section{ZDS.}

For small systems, there is no obvious threefold degeneracy at zero width while the threefold degeneracy is clearly seen when finite thickness is included ${ }^{56}$. For the largest system we have been able to study i.e. $N=16$, the picture becomes clearer. For $N=16$ electrons interacting via the pure Coulomb potential in the SLL we find that there is a threefold quasidegenerate set of ground states with quantum numbers of the Pfaffian for aspect ratio 0.99: see Fig. 11(a). However, we do not observe candidate, higher-lying, states forming their $\mathrm{p}$-h doublets that are expected from the higher energy combination of Pfaffian-Anti-Pfaffian states. If we tune the aspect ratio from 0.99 to 0.75 , then the three quasidegenerate ground states are still obtained but there is a less clear separation 

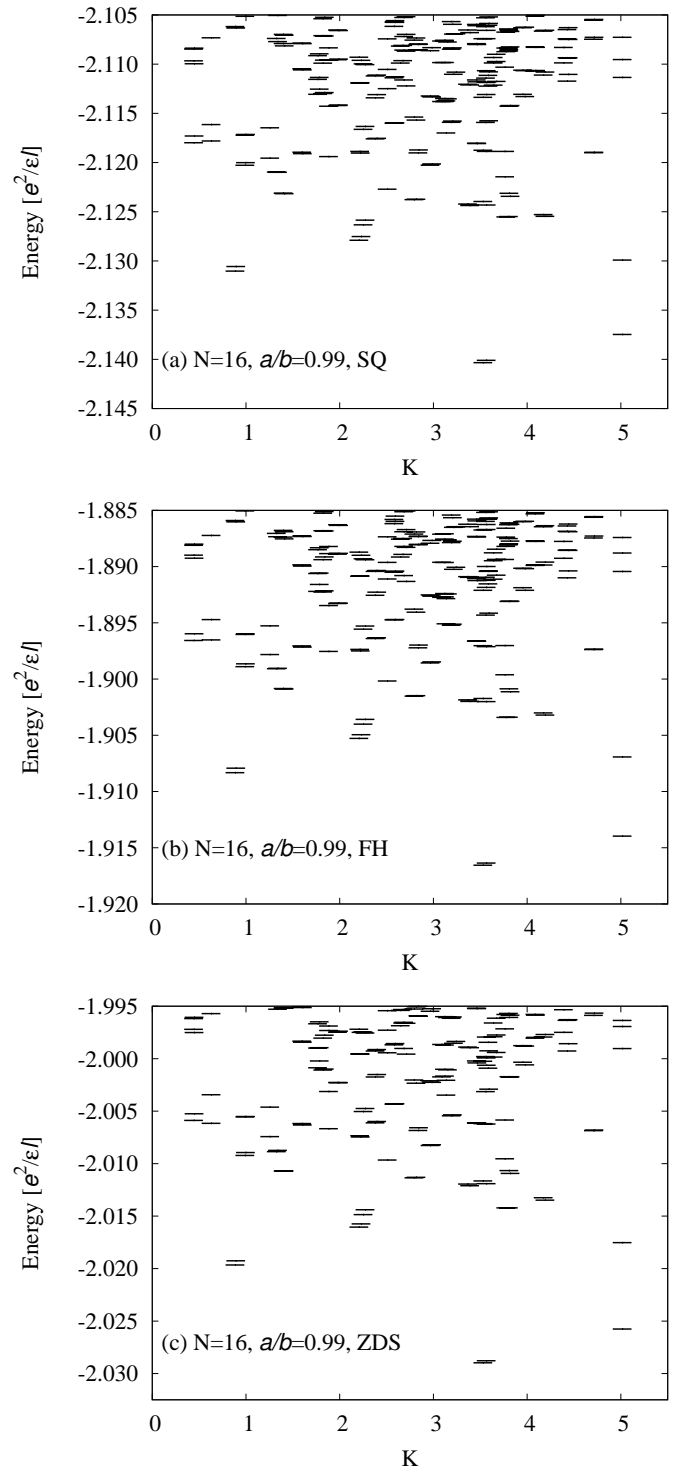

FIG. 13: Same as Fig. 12 except the aspect ratio has been tuned to 0.99 .

from the higher-lying states, see Fig. 11(b).

If we now consider systems with a finite width taken to be the value of maximum overlap $(d / l \sim 4-5)$, as determined from the calculations on the sphere, then we find the Pfaffian signature (threefold degeneracy and p$\mathrm{h}$ partner states) is qualitatively enhanced. At an aspect ratio equal to 0.75 the three finite thickness models considered all lead to the threefold quasidegeneracy for the three states with the correct quantum numbers: see Figs. 12(a)-(c). Now it is plausible to identify the $\mathrm{p}$-h partners for each of the states with the same quantum numbers predicted by the Moore-Read wavefunction slightly higher in energy than the ones forming the threefold quasidegenerate ground state manifold. If the aspect ratio, with finite thickness included, is changed from 0.75 to 0.99 we find that things change somewhat, see Figs.13(a)-(c); while there is still a threefold quasidegeneracy of the ground state, the doublet structure is no longer clear. The reason for this sensitivity to the aspect ratio is not known and deserves further study. We emphasize, however, that even if the enhancement of the Pfaffian signature is restricted to some range of aspect ratio, its presence is clearly enhanced by finite thickness.

The results presented in this subsection, taken with those in Sec. IIIA, provide a satisfying picture where finite thickness effects produce a ground state that is described by the MR Pfaffian wavefunction. The fact that two different geometries produce the same qualitative conclusion using two different, complementary, signatures is quite convincing. On the sphere (Sec. IIIA and Figs. (7. 9) we find that the overlap between the Pfaffian and the exact finite-system numerical wavefunction for the $\nu=5 / 2$ state is enhanced substantially as the quasi-2D thickness parameter is increased. On the torus (Sec. IIIB and Figs. 11]13) we find that the expected non-Abelian topological degeneracy, a characteristic signature for both Pfaffian/anti-Pfaffian states, shows up precisely where the wavefunction overlap is large.

\section{In-Plane magnetic field effects: Overlaps}

The consequence of the application of an in-plane magnetic field to the $\nu=5 / 2 \mathrm{FQH}$ state is a very important question. Experiments have shown ${ }^{81,82}$ that the FQHE at $\nu=5 / 2$ is suppressed with increasing in-plane component of the magnetic field. Originally, this was thought to point towards a spin-unpolarized FQH state at $5 / 2$, since traditionally the in-plane field is assumed to couple only to the spin degrees of freedom, and increasing the in-plane field is supposed to enhance the spinpolarization of the system. However, subsequent theoretical work ${ }^{37}$ seemed to settle the debate regarding the spin-polarization of the $5 / 2$ state, and the $5 / 2$ state is now considered to be spin-polarized. A question, therefore, arises about the suppression of the 5/2 FQHE induced by the finite in-plane field since spin-polarization presumably cannot play a role in a spin-polarized FQH state. It is speculated that the suppression arises from the in-plane field induced orbital effects leading to modifications of the pseudopotentials $V_{m}$.

The in-plane field serves to squeeze the single-particle electron wavefunction in the direction perpendicular to the plane. Thus, the application of an in-plane field serves to effectively decrease the width of the quantum well. From the previous section (i.e., section IIIA) it is clear that such a reduction in the effective width could lead to the destruction of the FQHE at $\nu=5 / 2$ and $7 / 3$ whereas it could seemingly strengthen the FQHE at $\nu=11 / 5$.

We now explore this in detail. Here we find that a parabolic confinement model is convenient, i.e., the $z$ direction confinement potential is $V(z)=\left(2 \hbar^{2} / m d_{0}^{4}\right) z^{2}$ where $d_{0}$ is the thickness of the quantum well. (In this 


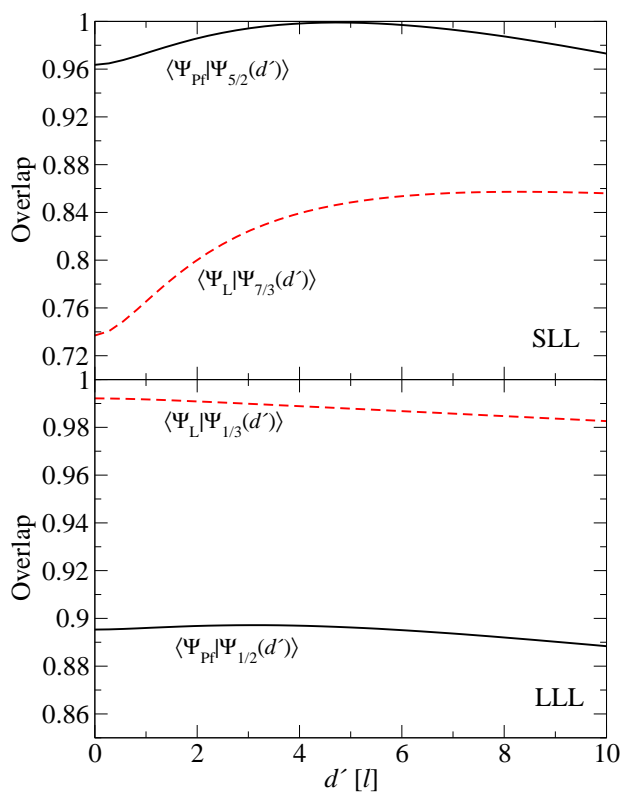

FIG. 14: (Color online) Overlap between the exact ground state wavefunction using a parabolic confinement at filling factor $1 / 3$ (dashed line) and 1/2 (solid line) and the Laughlin and Pfaffian wavefunction, respectively, as a function of $d^{\prime}$, in the SLL (top panel) and the LLL (bottom panel).

section we assume that the $d_{0}$ of the actual system is such that the overlap between the exact ground state and the Pfaffian wavefunction is maximum, that is, $d_{0}$ is a constant and not a variable, cf. section IIIA3,) The single-particle ground state electron wavefunction in the $z$-direction is thus a Gaussian

$$
\eta(z)=\left(\frac{2}{\pi d_{0}^{2}}\right)^{\frac{1}{4}} e^{-z^{2} / d_{0}^{2}} .
$$

We then apply an in-plane magnetic field of strength $B_{i}$ with the vector potential $\vec{A}_{i}=\left(B_{i} z, 0,0\right)$ and now make a simplifying assumption, namely, we ignore the cross terms (i.e., we consider the limit $B_{i} \ll B$ where $B$ is the perpendicular magnetic field strength) thus arriving at a slight modification, from the original quantum confinement, of the Schrodinger equation for $\eta(z)$ as

$$
\left[\frac{\hat{p}_{z}^{2}}{2 m}+V(z)+\frac{2 \hbar^{2}}{m} \frac{1}{4 l_{i}^{4}}\right] \eta(z)=E \eta(z)
$$

where $l_{i}^{2}=\hbar c / e B_{i}$. This equation has a solution of the same form as Eq. 15, i.e.,

$$
\eta(z)=\left(\frac{2}{\pi d^{2}}\right)^{\frac{1}{4}} e^{-z^{2} / d^{2}}
$$

with $1 / d^{4}=1 / d_{0}^{4}+1 / 4 l_{i}^{4}$. This implies that the quasi-2D layer thickness $d$ in the presence of $B_{i} \neq 0$ is less than the original thickness $d_{0}>d$, i.e., the $2 \mathrm{D}$ layer is squeezed by $B_{i}$.

The potential used when calculating the planar pseudopotentials is

$$
\tilde{V}(q)=\frac{e^{2}}{\epsilon} \frac{\operatorname{erfc}(q d / 2) e^{(q d)^{2} / 4}}{q},
$$

which includes the effects of a parabolic quantum well confinement and an in-plane magnetic field through the definition of $d$ above. Note that the effective $B_{i}$ dependent apparent width $d$ decreases as $B_{i}$ increases, squeezing the layer width parameter in the presence of the in-plane field.

The parabolic confinement is qualitatively similar to the confinement models used previously (SQ, FH, ZDS) and, in fact, quantitatively almost identical to the FangHoward confinement (see Ref. 37). In Fig. 14 we show the calculated overlap between the exact ground state wavefunction for $1 / 3$ and $1 / 2$ in the LLL and SLL with the Laughlin and Pfaffian wavefunction, respectively, as a function of $d^{\prime}$, to convince the reader that the difference between using a parabolic confinement and the FangHoward confinement is very small provided $d^{\prime}$ is defined as it was in section III A (here $d^{\prime}=(0.5 / 0.180756) d$ scaling it again with respect to the square-well confinement).

Obviously, $V(k) / d$ vs. $k d, V_{m}$ vs. $d$, and $f$ - and $g$ functions vs. $d$ (cf. Figs. 1, 3, 4, and 5) are very similar to the results shown for the Fang-Howard confinement and are, therefore, not shown.

To investigate the effect of a finite in-plane magnetic field we find it convenient to plot overlap as a function of the dimensionless variable $\lambda=\left(B_{i} / B\right)$ between the range $0<\lambda<2$. This variable is related to the effective thickness $d$ as $\lambda=2 \sqrt{1 / d^{4}-1 / d_{0}^{4}}$, where $d_{0}$ is chosen to be the value of thickness (without any in-plane magnetic field) at which the overlap is the highest, i.e., $d_{0} / l \sim 5$.

Finally, in Fig. 15 we plot the overlap between the exact ground state wavefunction for a parabolic confinement at a finite in-plane magnetic field $B_{i}$ and the Pfaffian wavefunction for $1 / 2$ in the SLL $(\nu=5 / 2)$, the Laughlin wavefunction at $1 / 3$ in the SLL $(\nu=7 / 3)$, and the Laughlin wavefunction at $1 / 3$ in the LLL $(\nu=1 / 3)$, respectively. The application of an in-plane magnetic field (i.e., $B_{i}, \lambda \neq 0$ ) causes the exact state at $\nu=5 / 2$ or $7 / 3$ to become more unlike the Pfaffian or Laughlin state, respectively, since the overlap goes down monotonically with increasing $B_{i} / B$, and hence, the in-plane field could eventually destroy the FQH at $\nu=5 / 2$ and $7 / 3$ by effectively enhancing the confinement, making the system more two-dimensional. On the other hand, the in-plane field causes the Laughlin state to become a better candidate for the exact state at $1 / 3$ since it makes the system more $2 \mathrm{D}$ (this is also true for $\nu=1 / 5$ in the LLL and SLL, which is not shown). Note that all of our in-plane field results follow directly from our findings in Sec. III A where we showed that increasing the layer width stabilizes the Pfaffian 5/2 and the Laughlin 7/3 states whereas it destabilizes the Laughlin $1 / 3$ state. In this section, we 


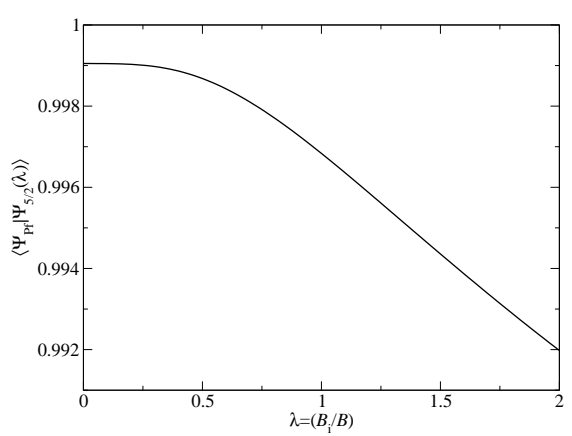

$\operatorname{SLL}(\nu=5 / 2)$

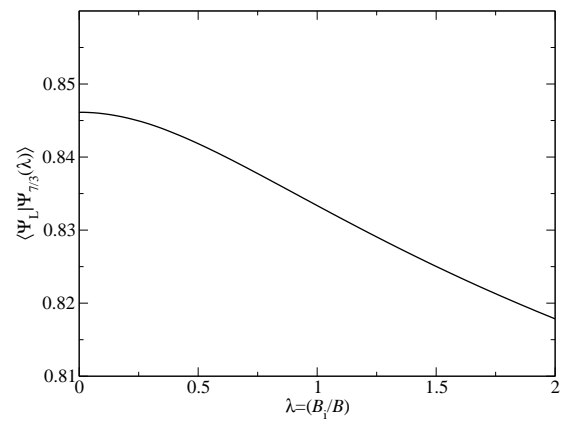

$\operatorname{SLL}(\nu=7 / 3)$

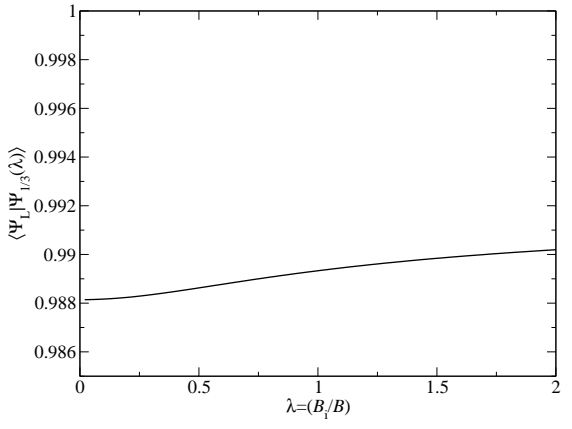

$\operatorname{LLL}(\nu=1 / 3)$

FIG. 15: Overlap between Pfaffian and the exact ground state wavefunctions at $5 / 2$ (left), the Laughlin and the exact wavefunctions at $7 / 3$ (middle), and the Laughlin and the exact wavefunctions at $1 / 3$ (right) as a function of $\lambda$.

explicitly show that an applied parallel field, by squeezing the quasi-2D layer width, could suppress the $5 / 2$ and $7 / 3$ states but strengthen the $1 / 3,1 / 5$, and $11 / 5$ states.

As important word of caution is, however, in order with respect to our in-plane field results presented in this section. We have only considered one particular aspect of the applied in-plane field, namely, the quasi2D confinement effect through Eqs. 15, 18, There are several additional effects induced by the in-plane field, which, although not considered in our work, may very well be important in practice. Two obvious spin effects of the in-plane field, neglected in our work because we are only considering completely spin-polarized states, are the Zeeman coupling induced spin polarization of the ground (and possibly excited) states. More subtle magneto-orbital effects neglected in our work are the in-plane field induced orbital anisotropy in the 2D plane and the subband-Landau level coupling induced enhanced scattering due to the finite in-plane fields, and the possible in-plane field induced stabilization of competing compressible states (e.g., stripe or bubble phases) which may have lower ground state energies than the incompressible FQHE states under our exclusive consideration in this work. A full consideration of all possible effects of the in-plane magnetic field is well beyond the scope of this paper, where we have concentrated entirely on the wavefunction overlap effect for FQH states arising from the wavefunction squeezing (i.e., $d<d_{0}$ ) by the applied in-plane field. In real experimental situations, some of these neglected effects may very well be significant or perhaps even dominant.

\section{CONNECTION TO PRIOR WORK}

The main question that we address in this section is the following. Do the first few Haldane pseudopotentials determine the FQHE physics in the SLL? This has been earlier studied in the literature, and therefore this section connects our work to the existing work of Morf 34 and Rezayi and Haldane ${ }^{35}$, which have been influential in theoretical studies of the $5 / 2$ state during the last 10 years.

As mentioned in the Introduction (Sec. II), early important work was done by Morf ${ }^{34}$ and Rezayi and Haldane ${ }^{35}$ regarding the Pfaffian description of the 5/2 FQHE. In particular, the overlap between the exact ground state of the SLL Coulomb potential, where the first pseudopotential $V_{1}^{(1)}$ was varied around the Coulomb point, and the Pfaffian was calculated as a function of this variation of the pseudopotential, i.e., $\delta V_{1}^{(1)}$. Rezayi-Haldane utilized the torus geometry varying both $V_{1}^{(1)}$ and $V_{3}^{(1)}$, and as such, their results are not directly comparable to the results presented here. Morf, however, varied $V_{1}^{(1)}$ using the spherical geometry and his results are directly applicable to ours. In fact, Fig.3(b) in Ref. 34 is equivalent to our Fig. 6(b) (middle plot)-of course, we have also included overlaps with respective Laughlin states as well as considered two (LLL and TLL) other LLs. A general result of Refs. 34 and 35 was that a positive $\delta V_{1}^{(1)}$ enhanced the overlap. However, as discussed in Sec. III A. for the Pfaffian, the change in overlap via the variation in the short range pseudopotentials is not easily motivated since the Pfaffian is not an exact eigenstate of a $V_{1}$-only two-body Hamiltonian, and, in fact, an increase in $V_{1}^{(1)}$ (leaving all other $V_{m}^{(1)}$ 's constant) is physically untenable, i.e., there is no experimental or physical way one can effect an increase only in $V_{1}^{(1)}$ in real systems.

Here we show that the first few pseudopotentials do not by themselves determine the physics. In Figs. 16, 17, and 18 we show the calculated overlap between the Pfaffian wavefunction and the exact ground state wavefunction of the SLL Coulomb Hamiltonian where $\left(V_{1}^{(1)}, V_{3}^{(1)}\right)$, $\left(V_{1}^{(1)}, V_{5}^{(1)}\right)$, and $\left(V_{3}^{(1)}, V_{5}^{(1)}\right)$ are allowed to vary away from their original SLL values by $\delta V_{m}^{(1)}$ for $N=8$ electrons and $2(Q+n)=13$, i.e., for the $5 / 2$ state. Each plot has left and right panels. The left panel is a color contour plot of the overlap as a function of the change in pseudopotentials. The right panel displays the same contour plot but with only contour lines at values of the 


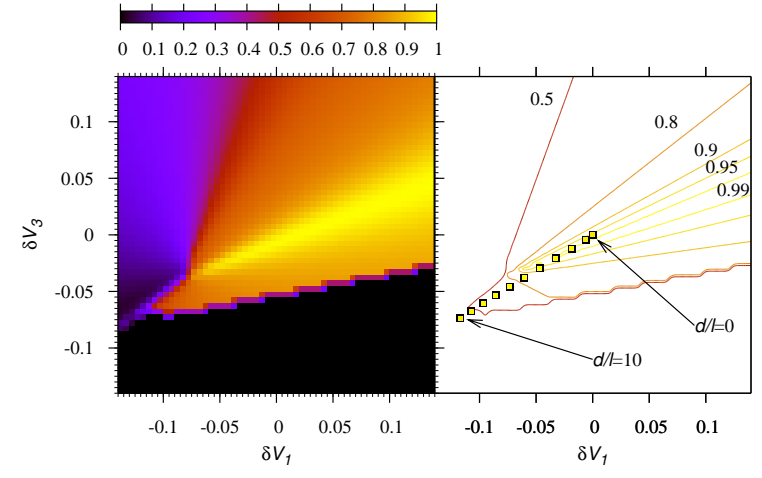

FIG. 16: (Color online) Contour plot of the overlap between the Pfaffian wavefunction and the $5 / 2$ ground state wavefunction of a SLL Coulomb Hamiltonian, where $V_{1}^{(1)}$ and $V_{3}^{(1)}$ have been varied from their pure Coulombic values, as a function of the variations $\delta V_{1}^{(1)}$ and $\delta V_{3}^{(1)}$. The system considered is the $N=8$ electron system shown in Fig. 9] The left panel is the color contour plot while the right panel contains only contour lines at values of the overlap equal to $0.5,0.8,0.9$, 0.95, and 0.99. Also shown in the left panel is the path traced out in the $\left(V_{1}^{(1)}, V_{3}^{(1)}\right)$ plane of the finite thickness of the SQ potential from $d / l=0$ to 10 .

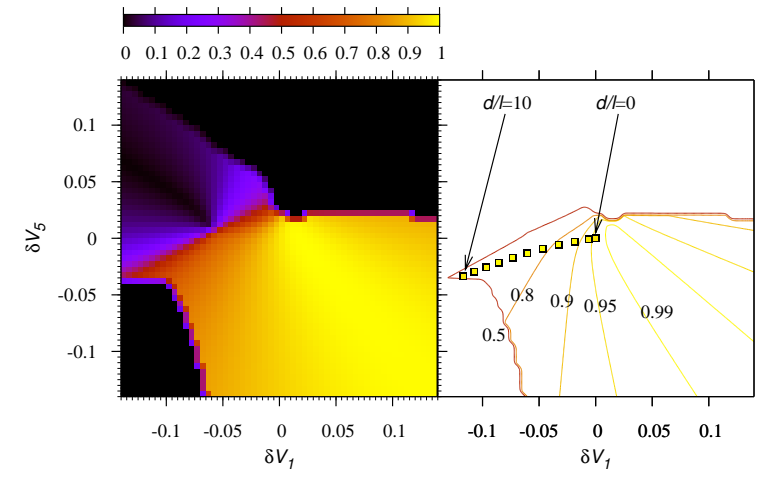

FIG. 17: (Color online) Same as Fig. 16 expect here we consider variations in $V_{1}^{(1)}$ and $V_{5}^{(1)}$.

overlap equal to $0.5,0.8,0.9,0.95$, and 0.99 . Also on this panel is a series of square points that show the overlap for the SQ potential at different values of $d / l$ (we use $d$ here since $d^{\prime}=d$ for the SQ by definition) from zero to $d / l=10$ in unit steps. Finite thickness changes all the $V_{m}^{(1)}$ 's but we are only showing the path traced out in the $V_{1}^{(1)}-V_{3}^{(1)}$ or $V_{1}^{(1)}-V_{5}^{(1)}$ or $V_{3}^{(1)}-V_{5}^{(1)}$ pseudopotential space of the contour plot.

Two main observations can be made in the parameters

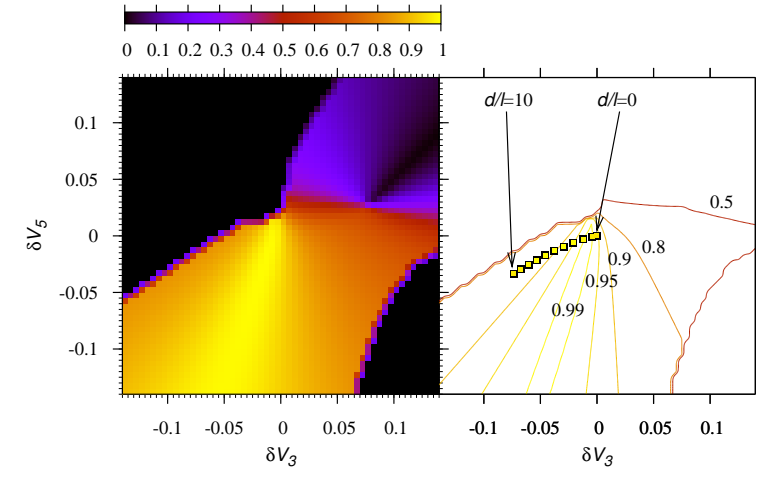

FIG. 18: (Color online) Same as Fig. 16] expect here we consider variations in $V_{3}^{(1)}$ and $V_{5}^{(1)}$.

space of $\left(V_{1}^{(1)}, V_{3}^{(1)}\right),\left(V_{1}^{(1)}, V_{5}^{(1)}\right)$, and $\left(V_{3}^{(1)}, V_{5}^{(1)}\right)$. First, there are always regimes where the overlap is above 0.99 . That this occurs in the $\left(V_{1}^{(1)}, V_{3}^{(1)}\right)$ space is not particularly surprising considering the previous work of RezayiHaldane $^{35}$ (although this is shown here for the first time in the spherical geometry). However, the other two contour plots in the $\left(V_{1}^{(1)}, V_{5}^{(1)}\right)$ and $\left(V_{3}^{(1)}, V_{5}^{(1)}\right)$ spaces go against the conventional wisdom. In particular, Fig. 18 shows that a large overlap with the Pfaffian can be obtained by leaving $V_{1}^{(1)}$ constant and varying $V_{3}^{(1)}$ and $V_{5}^{(1)}$ only. Hence, it is clear that the first two pseudopotentials do not dominate the physics of the 5/2 FQHE.

The second observation that can be gleaned is that, since the values of all the pseudopotentials change upon including finite thickness effects, one cannot parameterize finite thickness corrections in terms of only two pseudopotentials, be they the first and third, first and fifth, third and fifth, or any other combinations of two. This effect is clearly shown in the right panel of each contour plot where the path of the overlap, as a function of finite width, in the $\left(V_{1}^{(1)}, V_{3}^{(1)}\right),\left(V_{1}^{(1)}, V_{5}^{(1)}\right)$, and $\left(V_{3}^{(1)}, V_{5}^{(1)}\right)$ plane, respectively, does not coincide with a high overlap region shown in the contour plot when only changing two pseudopotentials. Note that the squares of the finite thickness overlap results are shaded according to the color contour plot on the left panel (as well as being more clearly visible in Fig. 91).

We have thus demonstrated that the investigation of the overlap as a function of changes (or ratios) between two pseudopotentials does not elucidate the physics of the $5 / 2$ state and produces ambiguous results. In particular, in the inset of Fig.5 of Rezayi-Haldane 35 the path of the finite thickness is plotted in the $\left(V_{1}^{(1)}, V_{3}^{(1)}\right)$ plane and shown to cross a boundary between a striped state and a paired FQHE state. However, from the above considerations, one cannot conclude that the effects of fi- 
nite thickness drive the system across boundaries in this way. Specifically, finite thickness corrections involve variations in all the pseudopotentials from the ideal Coulomb point, and tuning one or even a few pseudopotentials does not, under any circumstances, mimic the finite thickness effect. Tuning one or two pseudopotentials around the Coulomb point in order to study the stability of the $5 / 2$ FQHE state with respect to the Pfaffian is, therefore, somewhat misleading in our opinion, particularly since, unlike the LLL Laughlin states, there is no theoretical two-body Hamiltonian for which the Pfaffian is an exact ground state.

\section{FINITE THICKNESS EFFECTS ON EXCITATION GAPS}

The FQHE transport activation gap can be readily measured experimentally and is an extremely relevant quantity that characterizes the incompressibility of a FQH state. In this section we discuss the effects of finite thickness on the excitation gaps for the FQHE at $\nu=7 / 3$ and $5 / 2$. Usually the experimental activation gap and the theoretical excitation gap are considered to be the same although this may not be necessarily true in the presence of disorder. It is, however, well-accepted that larger FQHE gaps imply stronger FQH states associated with larger FQHE excitation energies.

For finite size systems there are a few ways to calculate the gap and we only consider the gap in the spherical geometry. The gap is considered to be the energy of a well separated quasiparticle-quasihole pair where the initial ground state is incompressible. Hence, one only considers the excitation gap if the ground state of the $N$ particle system at flux $Q$ is a uniform state with total angular momentum $L=0$. If this is the case then the excitation gap can be calculated as the energy of a quasiparticle $E_{0}^{(Q-1 / 2)}$ and a quasihole $E_{0}^{(Q+1 / 2)}$ where $E_{0}^{(Q)}$ is the ground state energy of a system of $N$ electrons at flux $Q$-hence, the quasihole state has $Q+1 / 2$ while the quasiparticle has $Q-1 / 2$. With these energies the gap is given as $\Delta=E_{0}^{(Q+1 / 2)}+E_{0}^{(Q-1 / 2)}-2 E_{0}^{(Q)}$. Note that this definition involves only calculations of ground state energies albeit at different flux values.

Another way to find the gap is to calculate the energy of the $N$ electron system at flux $Q$ as a function of angular momentum $L$. The ground state (again only if the state is uniform and thus incompressible) will have $L=0$, and a branch of low energy excited states at different $L \neq 0$. The gap is then given as the energy in the long wavelength limit which corresponds to the lowest energy excitation with $L=N$ for the state at $\nu=7 / 3$ and $L=N / 2$ for the, presumably, paired state at $\nu=5 / 2$. In other words,

$$
\Delta=E^{(Q)}\left(L_{e x}\right)-E^{(Q)}(L=0),
$$

where $L_{e x}=N(N / 2)$ for filling factor $7 / 3(5 / 2)$. This definition involves obtaining the low-energy spectra of

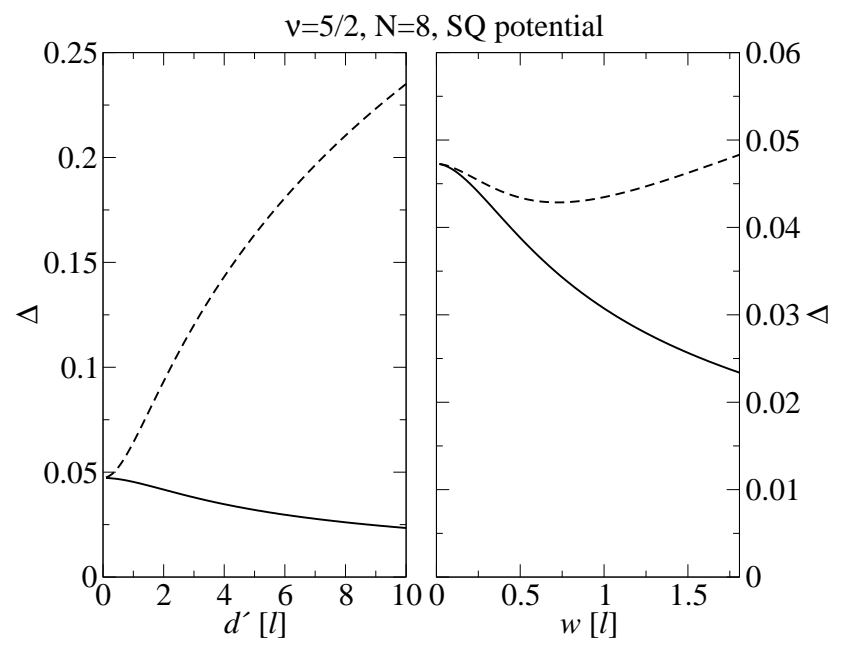

FIG. 19: Energy gap $\Delta$ for $\nu=5 / 2$ using the SQ potential as a function of $d^{\prime} / l$ (left) and $w / l$ (right). The solid line is the gap in units of $e^{2} / \epsilon l$ while the dashed line is the gap in units of $e^{2} / \epsilon \sqrt{l^{2}+d^{\prime 2}}$ (left) and $e^{2} / \epsilon \sqrt{l^{2}+w^{2}}$ (right). The system considered has $N=8$ electrons.

the system at a given $Q$. This is the method we use in this work to investigate the gap. The reason is that, as discussed by Morf ${ }^{37}$ and in Ref. 56, the first method, described above, leads to some ambiguity. This is because for $\nu=5 / 2$ and $N=8$ electrons the quasiparticle state is aliased to a FQHE state with filling factor $2 / 3$ in the SLL. Thus, what is being assumed as the quasiparticle energy from the $5 / 2 \mathrm{FQH}$ state may in fact be a $2 / 3$ filled incompressible FQH state. Since we are also not calculating the gap in the thermodynamic limit, it is not particularly important which method we use since we are only interested in broad qualitative features. See Refs. 37 and 44 for more thorough numerical investigations of the energy gaps in the FQHE. We emphasize that our results are only qualitative and should not be compared quantitatively with experimental activation gaps.

In Figs. 19 and 20 we plot the calculated gap for $\nu=5 / 2$ and $7 / 3$ for the SQ model for systems with $N=8$ and 6 electrons, respectively. The left panel shows the gap versus the width $d^{\prime}$ and the right panel shows the gap versus $w$, both in units of $l$. The solid line is the gap $\Delta$ in units of $e^{2} / \epsilon l$, and we see that for both filling factors the gap (in units of $e^{2} / \epsilon l$ ) decreases with increasing width (in units of $l$ ). This is not surprising since the scale of the energy itself is being reduced as the Coulomb energy is suppressed below $e^{2} / \epsilon l$ in the presence of finite thickness, e.g., see Fig. 3 where the pseudopotentials themselves are shown to decrease with increasing width. In fact, this introduces a subtle point when considering the theoretical energy gap including finite thickness effects. For every value of $d^{\prime}$ we are, essentially, considering a different Hamiltonian, so it is not quite appropriate to think of the gap as a function of the well width. This is most clear when one considers that the energy scale itself 


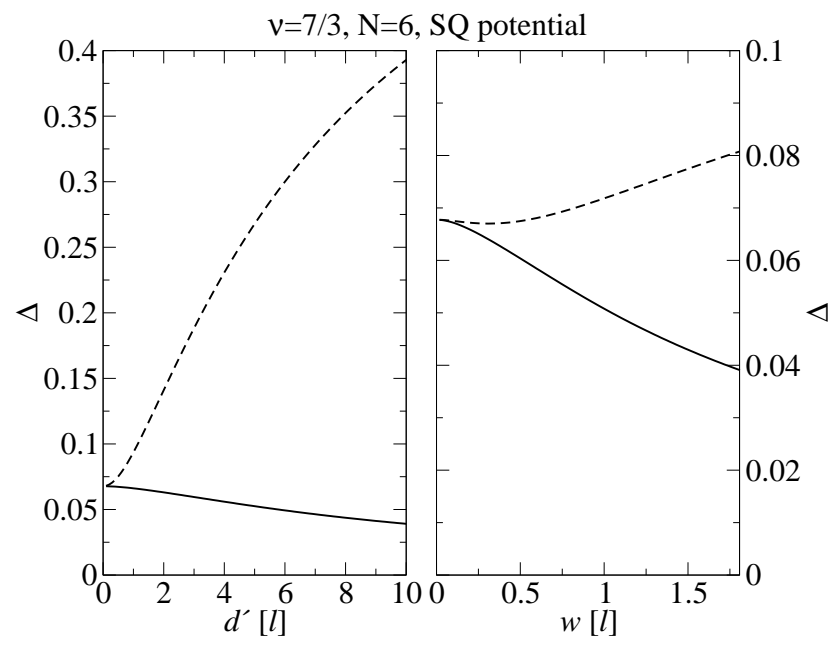

FIG. 20: Same as Fig. 19 except for $\nu=7 / 3$ and a system of $N=6$ electrons.

is changing with $d^{\prime}$. To readily incorporate the effect of a varying energy scale with varying $d^{\prime}$ we should rescale the gap energy. The dashed lines in the left (right) panels of Figs. 19 and 20] show the gap in units of $e^{2} / \epsilon \sqrt{l^{2}+d^{\prime 2}}$ $\left(e^{2} / \epsilon \sqrt{l^{2}+w^{2}}\right)$. In these units the gap is seen to increase with increasing width, i.e., in reduced energy units, the excitation gap is enhanced with increasing width.

We note that two alternative, and not necessarily equivalent, signatures exist for the theoretical characterization of the stability or the strength or the robustness of FQH incompressible states. These are (1) the overlap between the ground state finite-size numerical wavefunction and a candidate incompressible state, and, (2) the FQH excitation gap calculated directly numerically. The relationship between these two signatures of incompressibility is not obvious at all, and both definitions have their problems. The definition of the excitation gap is ambiguous (particularly due to the aliasing problem) since the two ways of defining it, as discussed above (using the ground state energy at different $Q$ and the excitation spectra at the same $Q$ ), are inequivalent. Also, a finite size system always has discrete energy levels, and thus always has a gap. In addition, the excitation energy $\Delta$, being an energy, is by definition not dimensionless, and therefore is somewhat problematic as a signature for the ground state compressibility. (For reasons discussed above, we believe that the widespread practice of expressing $\Delta$ in units of $e^{2} / \epsilon l$, the so-called Coulomb energy, is arbitrary, and $\left(\Delta /\left(e^{2} / \epsilon l\right)\right)$ may not necessarily be a faithful representation of the stability of ground state incompressibility, particularly in situations, e.g., $5 / 2$ state, where finite quasi-2D width is essential for the existence of the FQHE.) On the other hand, the signature of the overlap with a candidate analytical incompressible state (i.e., a variational ansatz such as the Laughlin or the MR wavefunction) has several intrinsic problems: (1) such an analysis is necessarily limited by the constraint of the variational ansatz, i.e., if a different (unknown) incompressible state describes the ground state better, the overlap calculation would miss that; (2) finite-size effects inherent in small system calculations, where a large overlap may turn out to be a misleading finite-size artifact; (3) the competition with nearby compressible states is not captured.

It is our contention that when one investigates the FQHE theoretically at some filling factor $\nu$ it is most illuminating to first determine the nature of the state responsible for the FQHE. This is usually done via an overlap calculation with some trial wavefunction (Laughlin, composite fermion, MR Pfaffian, etc.) such as was done in Sec. IIIA. Once a satisfying identification is made for the FQH ground state using the wavefunction overlap signature then experimentally relevant quantities, such as the excitation gap, can be calculated and compared with experiment. In fact, this is essentially the historical record of events following the discovery of the $\nu=1 / 3$ FQHE and subsequent explanation by Laughlin. This is also the strategy we have followed in the current work.

\section{CONCLUSION}

In this work, we investigate the relative stability of the FQHE at the most important primary filling factors $\nu=1 / 2,1 / 3$, and $1 / 5$ in the three lowest orbital Landau levels $n=0$ (LLL), 1 (SLL), and 2 (TLL) by calculating, as a function of the quasi-2D layer width parameter, the wavefunction overlap between the directly diagonalized exact many-body wavefunction (for small spherical systems) with the corresponding candidate theoretical ansatz for incompressible states, namely, the Laughlin wavefunction for $\nu=1 / 3$ and $1 / 5$ and the MR Pfaffian wavefunction for $\nu=1 / 2$. Rather surprisingly, we find the layer thickness parameter, often neglected in theoretical studies of the FQHE mostly carried out in the idealized zero-thickness strict $2 \mathrm{D}$ limit, to be a key parameter in stabilizing the incompressible states in the SLL. In particular, we find that the SLL states at $\nu=2+1 / 3=7 / 3$ and $2+1 / 2=5 / 2$ have larger exact overlap with the corresponding theoretical incompressible states for finite values of the layer thickness parameter $d^{\prime}$; typically, the overlap is maximum (of the order of unity, in fact) for $d^{\prime} / l \sim 4-5$ in the SLL whereas in the LLL the overlap decreases monotonically with increasing $d^{\prime} / l$, i.e., the incompressibility is the strongest at $d^{\prime}=0$ (note that the width parameter $d^{\prime} \propto d$ is the "normalized" width parameter, and $d^{\prime}=d$ only for the square-well confinement potential). Furthermore, we also find that finite width is essential to the observation of the threefold degenerate ground state signature of the MR Pfaffian state in the torus geometry. In particular, the expected threefold non-Abelian topological degeneracy for $\nu=5 / 2$ only shows up on the torus for $d^{\prime} \sim 4-5 l$, where the corresponding overlap with the MR wavefunction is optimal. 
In the TLL, the overlap is always (i.e., for any $d^{\prime} / l$ ) very small, indicating the essentially generic absence of incompressible FQH states in $n>1$ orbital LLs.

Our finding of the "absence" (i.e., small overlap) of the Laughlin $\nu=1 / 3$ state in the $d^{\prime}=0$ limit in the SLL is consistent with earlier theoretical work carried out in the ideal $d^{\prime}=0$ limit $47,48,49,69$. The experimental FQHE observations $\frac{12,13,14}{2}$ at $\nu=7 / 3$ and $8 / 3$ occur, of course, at finite values of $d^{\prime} / l$, and the experimental values of $d^{\prime} / l$ in the real samples is $d^{\prime} / l \sim 2-5$, which is in agreement with our theoretical finding. Our work indicates that tuning the quasi-2D width parameter significantly (either far above or below the optimal value where the overlap is maximum) should destroy incompressibility at $\nu=7 / 3$ (or $8 / 3)$.

The most important motivation for studying the SLL FQHE is to understand the nature of the enigmatic $5 / 2$ FQH state, the only even-denominator FQH state ever observed in single-layer 2D systems. What does our work imply for the stability of the anomalous FQHE at halffilling? We briefly discuss below the qualitative answer to this question.

Our work clearly shows the importance of the finite quasi-2D layer width in producing the incompressible FQH state at $\nu=5 / 2$, assuming the state to be described by the MR Pfaffian wavefunction which is the only available spin-polarized candidate wavefunction for the $5 / 2$ state. Consistent with earlier theoretical work in the literature ${ }^{34,35,39}$, we find the overlap between the exact (small-system) many-body wavefunction at $\nu=5 / 2$ to have a modest overlap $(\sim 0.8-0.9)$ with the Pfaffian wavefunction, which increases monotonically to a large overlap of almost unity as $d^{\prime} / l$ increases. For larger $d^{\prime} / l$, the overlap decreases again. Thus the behavior of the $5 / 2$ state as a function of finite width is similar to the $2+1 / 3=7 / 3$ Laughlin state-both are most stable at a finite layer width in contrast to the LLL Laughlin states at $1 / 3$ and $1 / 5$, which are most stable (i.e., maximum overlap $\sim 1)$ in the strict $2 \mathrm{D}\left(d^{\prime} / l=0\right)$ limit.

The behavior of the LLL $1 / 2$ state is interesting in this context. The overlap with the Pfaffian here shows a very slight increase as a function of $d^{\prime} / l$ before decreasing again similarly to the SLL 5/2 state except that the Pfaffian overlap never approaches unity for the LLL $1 / 2$ state, indicating that an incompressible $\nu=1 / 2 \mathrm{FQH}$ state, at least one that is well-described by the MR Pfaffian, is unlikely to occur in the LLL. We emphasize that there is no compelling fundamental reason for the LLL 1/2 state not manifesting a $\nu=1 / 2 \mathrm{FQHE}$, it is merely absent in the reasonable parameter space of the Coulomb interaction for realistic experimental systems. It is conceivable that a clever tuning of the interaction Hamiltonian far from the strict 2D Coulomb point will stabilize a LLL $\nu=1 / 2$ FQHE corresponding to the MR Pfaffian state.

The $\nu=1 / 5$ state in the SLL (i.e., $\nu=2+1 / 5=11 / 5$ ) is very similar to the LLL FQH states in its dependence on the layer width. The overlap with the Laughlin state decreases monotonically with increasing width parame- ter, implying that both the LLL and SLL 1/5 FQHE would be the strongest in the strict $2 \mathrm{D}$ ideal limit for zero layer thickness, similar to the situation for the $\nu=1 / 3$ LLL state.

Our work indicates that, in principle, wavefunction engineering should be possible to enhance the FQHE at $\nu=5 / 2$ and $7 / 3$ in the SLL by increasing the quasi$2 \mathrm{D}$ width parameter $d^{\prime} / l$. A trivial way of enhancing $d^{\prime} / l$ is, of course, to decrease $l$ (at fixed $d^{\prime}$ ) by increasing the magnetic field. Since increasing the magnetic field $B$ increases the interaction energy $\sim e^{2} / l \approx \sqrt{B}$, it is obvious that this would strengthen the FQHE trivially (the energy gap scales as $e^{2} / l$ thus increasing the gap). But increasing magnetic field while keeping $\nu$ fixed requires a proportional increase in the carrier density which is problematic (and may lead to the occupancy of the second subband, reducing the sample mobility substantially). Therefore, we suggest that $d^{\prime}$ should also be increased to produce enhanced stabilization of the SLL FQHE. The fact that different models of quasi-2D confinement, e.g., heterostructure (Fang-Howard), squarewell, parabolic quantum confinement, and the ZhangDas Sarma model, all give very similar qualitative and quantitative variation of our calculated overlap with the width parameter indicates that our qualitative conclusion is model independent, and applies to all quasi-2D physical systems where the FQHE is experimentally studied.

As a direct consequence of the layer width dependence of the FQHE found in this work, we also consider the experimentally important situation ${ }^{81,82}$ of the effect of an in-plane magnetic field, $B_{i}$, applied parallel to the quasi2D layer on the FQHE. Assuming the system to be completely spin-polarized, as we do throughout this work, and neglecting all disorder effects (i.e., ignoring for the moment that $B_{i}$ could cause additional scattering ${ }^{83,84}$ by inducing coupling between in-plane and transverse dynamics), the only effect of $B_{i}$ is to modify the quasi-2D layer width $d^{\prime}$ by shrinking it to $d_{i}^{\prime}\left(B_{i}\right)<d_{i}^{\prime}\left(B_{i}=0\right)$. This in-plane magnetic field induced modification of the quasi-2D layer width arises from the magneto-orbital coupling between in-plane and transverse electron dynamics in a finite-width system, which is strictly absent in the ideal 2D limit where, in the absence of spin (i.e., for a completely spin-polarized system), there can be no coupling between the in-plane and the transverse motion. We show that in a quasi-2D system, the in-plane field $B_{i}$ could destabilize the SLL 5/2 Pfaffian state by decreasing the effective layer width through the reduction of the overlap between the exact wavefunction and the Pfaffian wavefunction. This follows naturally from our finding that in the SLL, the reduction of the quasi-2D layer width always reduces the overlap of the $5 / 2$ state and since $B_{i}$ reduces the effective value of the layer width it would naturally suppress the overlap at $\nu=5 / 2$. The same is also true for $\nu=7 / 3$, but not for $\nu=11 / 5$ in the SLL or for $\nu=1 / 3$ or $1 / 5$ in the LLL since the latter three FQH states are the most robust (i.e., maximum overlap) at the smallest value of $d^{\prime}$. We therefore predict 
that, to the extent the $1 / 2,1 / 3$, and $1 / 5 \mathrm{FQH}$ states are completely spin-polarized, the application of a parallel magnetic field is likely to weaken the $\nu=5 / 2$ and $7 / 3$ FQH states and strengthen the $\nu=11 / 5,1 / 3$, and $1 / 5$ FQH states. Of course, the physical effects neglected in our approximation (e.g., spin, Landau level coupling, disorder) could play important roles in actual experiments, but we suggest careful experiments in an applied in-plane field to validate (or falsify) our prediction of the weakening (strengthening) of the $5 / 2,7 / 3(11 / 5)$ FQHE in the SLL. We mention that the quantitative effect of the in-plane field depends strongly on $l_{i} / l$ and $l_{i} / d^{\prime}$ where $l_{i} \equiv\left(c \hbar / e B_{i}\right)^{1 / 2}$ and $l$ and $d^{\prime}$ are the $2 \mathrm{D}$ magnetic length and the $B_{i}=0$ value of the width, respectively. Since $l_{i} / l$ and $l_{i} / d^{\prime}$ both should be not too large for the orbital influence of the in-plane field to be observable, it may not be easy to observe the predicted effect in the LLL (or even for the $11 / 5 \mathrm{SLL}$ ) since the typical "l" is rather small for these cases.

Before concluding we summarize the large number of approximations we have made in our theory: (1) We have assumed a spin-polarized system throughout, and therefore if spin is playing an important dynamical role in any of the fractional states we study, then our results would not be particularly useful in understanding the corresponding experimental observations. (2) We have neglected the Landau level coupling throughout our work, and it is possible that the LL coupling plays a role in the SLL FQHE ${ }^{85}$. (3) We have neglected all disorder effects-in particular, the application of the in-plane parallel field may introduce additional "disorder" by opening new channels of scattering (e.g., intersubband scattering) through the coupling of the in-plane and transverse dynamics. This will then serve to further weaken the $5 / 2$ and $7 / 3 \mathrm{FQHE}$ in the presence of the in-plane field. We expect such scattering effects to be very weak in the $l_{i} \gtrsim d^{\prime}$ and $l_{i} \gtrsim l$ regime of experimental interest. (4) We have uncritically assumed that the "strength" of the incompressibility at a given filling factor (or equivalently, the robustness of the FQHE at a given fraction) is determined by the calculated overlap between the exactly diagonalized numerical wavefunction for small system sizes and the corresponding candidate theoretically postulated FQH state, i.e., the Laughlin wavefunction for $\nu=1 / 5,1 / 3$ (in all LLs) and the MR Pfaffian wavefunction for $\nu=1 / 2$ (in all LLs). (5) Related to the last point, our work will completely fail if the actual state describing the FQHE at a particular fraction is qualitatively different from our assumed candidate wavefunctions. For example, one cannot, as a matter of principle, rule out the possibility, unlikely as it may seem, that the observed $\nu=5 / 2 \mathrm{FQH}$ state is the MR Pfaffian state for finite $d^{\prime} / l \sim 4-5$, as we find, which continuously and adiabatically goes over to some other unknown incompressible state at lower values of $d^{\prime} / l$. Such a continuous crossover implies that the observed 5/2 FQHE will remain strong and robust for all values of $d^{\prime}$, but our calculated overlap with just the Pfaffian decreases as $d^{\prime} / l$ decreases. We believe such a scenario to be extremely unlikely, particularly since there are no other proposed wavefunctions for $5 / 2$, but we cannot rule this out on purely theoretical grounds. (The same also applies for the $7 / 3$ state where, if anything, such a scenario of two distinct states, Laughlin for layer $d^{\prime} / l$ and "something else" for smaller $d^{\prime} / l$, seems even more unlikely.) (6) We have used approximate models for considering the finite layer width in the quasi-2D system. However, the fact that four distinct models of quasi-2D systems produce essentially identical results and conclusions indicate that our results should have qualitative and semi-quantitative validity in real samples. Also, earlier theoretical work ${ }^{21,22,37}$ indicates that more sophisticated models of quasi-2D confinement do not lead to appreciable changes in the FQHE numerical results compared with the relatively simple models used in our work. (7) We have utilized the standard spherical geometry for our finite size diagonalization using rather modest system sizes (number of electrons between 5 and 10 depending on the filling factor). We believe that the small system size of our exact diagonalization study is not a problem since we are not attempting any quantitative estimates of the excitation gap (or other experimental quantities), but are interested in the qualitative dependence of the overlap as a function of the layer width in different LLs. One reason for our use of relatively modest system size is, of course, computational ease (since we are producing a very large amount of numerical data: four different models of confinement, and three different LLs, for three different FQH states, i.e., 36 sets of diagonalization done as a function of the width parameter $d^{\prime}$ !). Yet a second important reason for our choice of system sizes is to avoid the well-known "aliasing" problem on the sphere where two distinct fractions occur together exactly at the same parameter values. We have only chosen system sizes where the aliasing issue does not arise. The fact that we study three different orbital LLs on an equal footing to compare the relative qualitative stability of the $1 / 3,1 / 5$ and $1 / 2$ states gives us confidence in our numerically obtained trends (as a function of $d^{\prime}$ ) and conclusions, if not the precise numbers.

Finally, we emphasize an important qualitative finding of our work. We find that, in contrast to the Laughlin states (e.g., $\nu=1 / 3$ ) in the LLL where increasing the first pseudopotential $V_{1}$ compared with the Coulomb value necessarily strengthens the FQHE in a theoretically and physically meaningful manner, no such simple pseudopotential adjustments (either a single pseudopotential, e.g., $V_{1}, V_{3}, V_{5}$ or arbitrary combinations of them, e.g., $\left.\left(V_{1}, V_{3}\right),\left(V_{1}, V_{5}\right),\left(V_{3}, V_{5}\right)\right)$ make sense for studying the FQHE stability in higher (SLL or TLL) orbital Landau levels. This is because of the theoretical fact that the $1 / q$ Laughlin state becomes and exact LLL eigenstate of the effective two-body Hamiltonian $\hat{H}_{L}^{(q)}$ (see Eq. 6) where all pseudopotentials $m \geq q$ are taken to be zero, e.g., the Laughlin $1 / 3$ state is just an exact LLL eigenstate of the Coulomb interaction if $V_{1} \rightarrow \infty$. This mathematical 
simplicity provides an adiabatic connection between the Laughlin state and the exact $\nu=1 / 3 \mathrm{FQH}$ state even in the realistic system as long as $V_{1}$ is not too "small". This mathematical simplicity is, however, completely lost in higher orbital LLs where the exact state cannot simply be written down in this manner. In particular, the $\nu=5 / 2$ even-denominator state in the SLL, if it is indeed the Moore-Read Pfaffian state, is not an eigenstate of any (even a completely unrealistic) two-body Hamiltonian. As such, trying to understand the nature of the $5 / 2$ state by varying the few lower pseudopotentials (e.g., $V_{1}$, $V_{3}$, etc.) around the Coulomb point is not mathematically (or physically) well-motivated since no two-body Hamiltonian exists with the MR state as its exact ground state. We have therefore adopted the physically motivated approach in this work by working directly with the realistic Hamiltonian including the quasi-2D finite thickness effects (instead of using unrealistic arbitrary variations in the pseudopotentials). Varying the finite quasi-2D layer thickness involves changes in all the pseudopotentials which cannot be mimicked by varying any one or two pseudopotentials only. We find that this phys- ical approach leads to a rather unexpected finding: While in the LLL, finite quasi-2D thickness always weakens the FQHE, in the SLL, the finite thickness may actually stabilize the FQHE, for example, at $\nu=5 / 2$ and $7 / 3$ filling factor.

In summary, we have theoretically considered the effect of orbital dynamics on the stability of the primary FQH states at $1 / 2,1 / 3$, and $1 / 5$ filling factors by calculating the wavefunction overlap (and the topological degeneracy expected for the MR Pfaffian state) as a function of the quasi-2D layer width $\left(d^{\prime}\right)$ in $n=0$ (LLL), 1 (SLL), and 2 (TLL) orbital LLs, finding that the FQHE does not occur in the TLL (for any value of the quasi-2D width parameter), is the most robust at $d^{\prime}=0$ in the LLL and for the $11 / 5$ state in the SLL, and is the most robust at $d^{\prime} / l \sim 4-5$ in the SLL for the $5 / 2$ and $7 / 3$ state. We also do not find any $\nu=1 / 2 \mathrm{FQHE}$ in the LLL for any value of the layer width.

MRP and SDS acknowledge support from the Microsoft Q Project. TJ acknowledges support from IFRAF. Numerical calculations have involved computer time allocation IDRIS-072124.
1 D. C. Tsui, H. L. Stormer, and A. C. Gossard, Phys. Rev. Lett. 48, 1559 (1982).

2 R. B. Laughlin, Phys. Rev. Lett. 50, 1395 (1983).

3 J. K. Jain, Phys. Rev. Lett. 63, 199 (1989).

4 B. I. Halperin, Phys. Rev. Lett. 52, 1583 (1984).

5 J. M. Leinaas and J. Myrheim, Nuovo Cim. B37, 1 (1977).

${ }_{7}^{6}$ F. Wilczek, Phys. Rev. Lett. 49, 957 (1982).

7 Perspectives in Quantum Hall Effects, edited by S. Das Sarma and A. Pinczuk (Wiley, New York, 1997).

8 The Quantum Hall Effect, edited by R. E. Prange and S. M. Girvin (Springer-Verlag, New York, 1990).

9 J. K. Jain, Composite Fermions (Cambridge University Press, Cambridge, 2007).

${ }^{10}$ R. Willett, J. P. Eisenstein, H. L. Störmer, D. C. Tsui, A. C. Gossard, and J. H. English, Phys. Rev. Lett. 59, 1776 (1987).

11 W. Pan, J.-S. Xia, V. Shvarts, D. E. Adams, H. L. Störmer, D. C. Tsui, L. N. Pfeiffer, K. W. Baldwin, and K. W. West, Phys. Rev. Lett. 83, 3530 (1999).

12 J. P. Eisenstein, K. B. Cooper, L. N. Pfeiffer, and K. W. West, Phys. Rev. Lett. 88, 076801 (2002).

13 J. S. Xia, W. Pan, C. L. Vicente, E. D. Adams, N. S. Sullivan, H. L. Störmer, D. C. Tsui, L. N. Pfeiffer, K. W. Baldwin, and K. W. West, Phys. Rev. Lett. 93, 176809 (2004).

14 G. A. Csathy, J. S. Xia, C. L. Vicente, E. D. Adams, N. S. Sullivan, H. L. Störmer, D. C. Tsui, L. N. Pfeiffer, and K. W. West, Phys. Rev. Lett. 94, 146801 (2005).

15 H. C. Choi, W. Kang, S. Das Sarma, L. N. Pfeiffer, and K. W. West, Phys. Rev. B 77, 081301 (2007).

${ }^{16}$ W. Pan, J. S. Xia, H. L. Störmer, D. C. Tsui, C. Vicente, E. D. Adams, N. S. Sullivan, L. N. Pfeiffer, K. W. Baldwin, and K. W. West, Phys. Rev. B 77, 075307 (2007).

17 G. Gervais, L. W. Engel, H. L. Störmer, D. C. Tsui, K. W. Baldwin, K. W. West, and L. N. Pfeiffer, Phys. Rev. Lett.
93, 266804 (2004).

18 A. H. MacDonald and G. C. Aers, Phys. Rev. B 29, 5976 (1984).

19 S. He, F. C. Zhang, X. C. Xie, and S. Das Sarma, Phys. Rev. B 42, 11376 (1990).

20 F. C. Zhang and S. Das Sarma, Phys. Rev. B 33, 2903 (1986).

21 M. W. Ortalano, S. He, and S. Das Sarma, Phys. Rev. B 55, 7702 (1997).

22 K. Park and J. K. Jain, Phys. Rev. Lett. 81, 4200 (1998).

${ }^{23}$ K. Park, N. Meskini, and J. K. Jain, Journal of Physics: Condensed Matter 11, 7283 (1999).

24 P. L. Gammel, D. J. Bishop, J. P. Eisenstein, J. H. English, A. C. Gossard, R. Ruel, and H. L. Störmer, Phys. Rev. B 38, 10128 (1988).

25 J. P. Eisenstein, R. Willett, H. L. Störmer, D. C. Tsui, A. C. Gossard, and J. H. English, Phys. Rev. Lett. 61, 997 (1988).

26 K. v. Klitzing, G. Dorda, and M. Pepper, Phys. Rev. Lett. 45, 494 (1980).

27 Y. W. Suen, L. W. Engel, M. B. Santos, M. Shayegan, and D. C. Tsui, Phys. Rev. Lett. 68, 1379 (1992).

28 J. P. Eisenstein, G. S. Boebinger, L. N. Pfeiffer, K. W. West, and Song He, Phys. Rev. Lett. 68, 1383 (1992).

29 J. P. Eisenstein in Ref. 7.

30 S. M. Girvin and A. H. MacDonald in Ref. 7.

31 Song He, S. Das Sarma, and X. C. Xie, Phys. Rev. B 47, 4394 (1993).

32 B. I. Halperin, Helv. Phys. Acta 56, 783 (1983).

33 G. Moore and N. Read, Nucl. Phys. B 360, 362 (1991).

${ }^{34}$ R. H. Morf, Phys. Rev. Lett. 80, 1505 (1998).

35 E. H. Rezayi and F. D. M. Haldane, Phys. Rev. Lett. 84, 4685 (2000).

36 N. Read and E. Rezayi, Phys. Rev. B 54, 16864 (1996).

37 R. H. Morf, N. d'Ambrumenil, and S. Das Sarma, Phys. 
Rev. B 66, 075408 (2002).

38 V. W. Scarola, K. Park, and J. K. Jain, Nature 406, 863 (2000).

39 V. W. Scarola, J. K. Jain, and E. H. Rezayi, Phys. Rev. Lett. 88, 216804 (2002).

${ }^{40}$ C. Toke and J. K. Jain, Phys. Rev. Lett. 96, 246805 (2006).

41 C. Toke, N. Regnault, and J. K. Jain, Phys. Rev. Lett. 98, 036806 (2007).

42 A. Wojs and J. J. Quinn, Phys. Rev. B 74, 235319 (2006).

43 G. Möller and S. H. Simon, Phys. Ref. B 77, 075319 (2008).

44 A. E. Feiguin, E. Rezayi, C. Nayak, and S. Das Sarma, Phys. Rev. Lett.100, 166803 (2008).

45 N. Read, Physica B: Condensed Matter 298, 121 (2001).

46 F. D. M. Haldane and E. H. Rezayi, Phys. Rev. Lett. 60, 956 (1988)

47 A. H. MacDonald, Phys. Rev. B 30, 3550 (1984).

48 C. Toke, M. R. Peterson, G. S. Jeon, and J. K. Jain, Phys. Rev. B 72, 125315 (2005).

49 N. d'Ambrumenil and A. M. Reynolds, J. Phys. C 21, 119 (1988).

50 V. W. Scarola, Kwon Park, and J. K. Jain, Phys. Rev. B 62, R16259 (2000).

51 S. Das Sarma, M. Freedman, and C. Nayak, Phys. Rev. Lett. 94, 166802 (2005).

52 C. Nayak, S. H. Simon, A. Stern, M. Freedman, and S. Das Sarma, arXiv:0707.1889v1 [cond-mat.str-el] (2007).

${ }^{53}$ R. L. Willett, M. J. Manfra, L. N. Pfeiffer, and K. W. West, arXiv:0807.0221 1 [cond-mat.mes-hall] (2008).

54 M. Dolev, M. Heiblum, V. Umansky, Ady Stern, and D. Mahalu, Nature 452, 829 (2008).

55 I. P. Radu, J. B. Miller, C. M. Marcus, M. A. Kastner, L. N. Pfeiffer, and K. W. West, Science 320, 899 (2008).

56 M. R. Peterson, Th. Jolicoeur, and S. Das Sarma, Phys. Rev. Lett. 101, 016807 (2008).

57 F. D. M. Haldane, Phys. Rev. Lett. 51, 605 (1983).

${ }^{58}$ F. D. M. Haldane, in Ref. 8 .

59 M. Greiter, X.-G. Wen, and F. Wilczek, Phys. Rev. Lett. 66, 3205 (1991).

60 S. Das Sarma and B. A. Mason, Ann. Phys. 163, 78 (1985).

${ }^{61}$ T. Ando, A. B. Fowler, and F. Stern, Rev. Mod. Phys. 54, 437 (1982).

${ }^{62}$ F. Stern and S. Das Sarma, Phys. Rev. B 30, 840 (1984).

${ }^{63}$ L. Belkhir and J. K. Jain, Solid State Commun. 94, 107 (1995).

64 T. T. Wu and C. N. Yang, Nucl. Phys. B 107, 365 (1976).

${ }^{65}$ G. Fano, F. Ortolani, and E. Colombo, Phys. Rev. B 34,
2670 (1986).

66 The single particle angular momentum $l$ is related to the monopole strength $Q$ through $l=Q+n$ in the spherical geometry.

67 Furthermore, we are not trying to break any records by diagonalizing the largest systems. The larger systems will be left to other works, i.e., Ref. 56

68 With increasing $d^{\prime}$ the ZDS potential gives an overlap that drops off significantly towards zero, cf. Ref. 19 .

69 T. Sbeouelji and N. Meskini, Phys. Rev. B 64, 193305 (2001).

70 B. I. Halperin, and P. A. Lee and N. Read, Phys. Rev. B 47, 7312 (1993).

71 F. D. M. Haldane, Phys. Rev. Lett. 55, 2095 (1985).

72 E. H. Rezayi, F. D. M. Haldane, and K. Yang, Phys. Rev. Lett. 83, 1219 (1999).

73 F. D. M. Haldane, E. H. Rezayi, and K. Yang, Phys. Rev. Lett. 85, 5396 (2000).

74 K. Yang, F. D. M. Haldane, and E. H. Rezayi, Phys. Rev. B64, 081301(R) (2001).

75 F. D. M. Haldane and E. H. Rezayi, Phys. Rev. B 31, 2529 (1985).

76 M. Greiter, X. G. Wen, and F. Wilczek, Nucl. Phys. B 374, 567 (1992).

77 S. B. Chung and M. Stone, J. Phys. A 40, 4923 (2007).

78 M. Levin, B. I. Halperin, and B. Rosenow, Phys. Rev. Lett. 99, 236806 (2007).

79 S.-S. Lee, S. Ryu, C. Nayak, and M. P. A. Fisher, Phys. Rev. Lett. 99, 236807 (2007).

80 M. R. Peterson, Kwon Park, and S. Das Sarma, arXiv:0807.0638v1 [cond-mat.mes-hall] (2008).

81 J. P. Eisenstein, R. Willett, H. L. Störmer, D. C. Tsui, A. C. Gossard, and J. H. English, Phys. Rev. Lett. 61, 997 (1988).

82 R. R. Du, A. S. Yeh, H. L. Störmer, D. C. Tsui, L. N. Pfeiffer, and K. W. West, Phys. Rev. Lett. 75, 3926 (1995).

83 E. H. Hwang and S. Das Sarma, Phys. Rev. B 73, 121309 (2006).

84 S. Das Sarma and E. H. Hwang, Phys. Rev. Lett. 84, 5596 (2000).

85 C. R. Dean, B. A. Piot, P. Hayden, S. Das Sarma, G. Gervais, L. N. Pfeiffer, and K. W. West, Phys. Rev. Lett. 100, 146803 (2008); ibid, arXiv:0805.3349 [cond-mat.meshall] (2008). 